ESAIM: M2AN

Vol. 40, No 4, 2006, pp. 735-764

DOI: $10.1051 / \mathrm{m} 2 \mathrm{an}: 2006032$
ESAIM: Mathematical Modelling and Numerical Analysis

www.edpsciences.org/m2an

\title{
THE APPROXIMATE RIEMANN SOLVER OF ROE APPLIED TO A DRIFT-FLUX TWO-PHASE FLOW MODEL
}

\author{
Tore Fli̊tten ${ }^{1,2}$ And Svend TOllak Munkejord ${ }^{3}$
}

\begin{abstract}
We construct a Roe-type numerical scheme for approximating the solutions of a driftflux two-phase flow model. The model incorporates a set of highly complex closure laws, and the fluxes are generally not algebraic functions of the conserved variables. Hence, the classical approach of constructing a Roe solver by means of parameter vectors is unfeasible. Alternative approaches for analytically constructing the Roe solver are discussed, and a formulation of the Roe solver valid for general closure laws is derived. In particular, a fully analytical Roe matrix is obtained for the special case of the Zuber-Findlay law describing bubbly flows. First and second-order accurate versions of the scheme are demonstrated by numerical examples.
\end{abstract}

Mathematics Subject Classification. 35L65, 76M12, 76T10.

Received: January 14, 2006. Revised: May 12, 2006.

\section{INTRODUCTION}

To avoid excessive computational complexity, workable models describing two-phase flows in pipe networks are conventionally obtained by means of some averaging procedure. Models thus obtained are mathematically tractable, but there is a significant loss of information associated with the averaging process.

Hence additional information must be supplied to the system in the form of closure laws. The different physical assumptions leading to such laws result in different formulations of the two-phase flow models $[1,19,21,26]$. It is useful to divide such models into two main classes:

- Two-fluid models, where equations are written for mass, momentum and energy balances for each fluid separately.

- Mixture models, where equations for the conservation of physical properties are written for the two-phase mixture.

For reasons of accuracy and robustness, it is desirable to use a numerical method able to provide an upwind resolution of all wave phenomena inherent in the models. The approximate Riemann solver of Roe [22] is a convenient candidate, as it requires only the solution of a linear Riemann problem at each cell interface.

Keywords and phrases. Two-phase flow, drift-flux model, Riemann solver, Roe scheme.

1 The International Research Institute of Stavanger, Prof. Olav Hanssensvei 15, Stavanger, Norway.

Tore.Flaatten@irisresearch.no

2 Current address: Centre of Mathematics for Applications, 1053 Blindern, 0316 Oslo, Norway.

3 Department of Energy and Process Engineering, Norwegian University of Science and Technology, Kolbjørn Hejes veg 1A,

7491 Trondheim, Norway. stm@pvv.ntnu.no

(c) EDP Sciences, SMAI 2006 
In the context of two-phase flows, this method has been extensively used. Sainsaulieu [24] proposed a Roetype Riemann solver for a model describing incompressible liquid droplets suspended in a gas. Karni et al. [15] implemented a Roe scheme for a two-fluid model with velocity and pressure relaxation [25].

A classical two-fluid model [26] assumes pressure equilibrium between the phases. Toumi and Kumbaro [31] presented a Roe scheme for such a model including a virtual mass force term. A generalization allowing for a pressure-modification term at the gas-liquid interface was presented in [29]. An alternative Roe scheme for this model was presented by Evje and Flåtten [9]. Furthermore, Cortes et al. [6] proposed an efficient method for calculating the wave structure of the Roe linearization for such a model.

In this paper, we consider a mixture model describing two-phase flows where the motions of the phases are strongly coupled. The model, commonly denoted as the drift-flux model, consists of a mass conservation equation for each phase, in addition to a momentum balance equation for the two-phase mixture. Supplementary relations are required to obtain the information necessary for determining the motion of each phase separately.

These relations are commonly expressed in terms of a hydrodynamic closure law giving the relative velocity between the phases as a function of the flow parameters:

$$
v_{\mathrm{g}}-v_{\ell}=\Phi\left(m_{\mathrm{g}}, m_{\ell}, v_{\mathrm{g}}\right)
$$

where $v_{k}$ is the velocity and $m_{k}$ is the volumetric mass of phase $k$. The relative velocity $v_{\mathrm{r}}=v_{\mathrm{g}}-v_{\ell}$ between the phases is often referred to as the slip velocity; for this reason, the closure law (1) is also commonly known as the slip relation.

In general, the closure law $\Phi$ is commonly stated as a complex combination of analytic expressions valid for particular flow regimes, experimental correlations, and various switching operators. To the investigator, it may be viewed as a black box. However, a very important special case is the Zuber-Findlay [33] slip relation which can be written in a simple analytical form. The applicability of the various hydrodynamic laws will be discussed in Section 1.1.3.

In addition, thermodynamic closure laws must be specified for each phase to relate the phasic density to the mixture pressure. These relations are often given only in tabular form.

As has been pointed out by several researchers $[2,3,7,8,10,23]$, the complexity of these laws severely restricts the possibilities for constructing a Roe solver by purely algebraic manipulations. Nevertheless, Roe-type schemes have been proposed for this model. Romate [23] presented a method for constructing a Roe matrix using a fully numerical approach. This method was used as the conservative part of the hybrid primitive-conservative method of Fjelde and Karlsen [11]. Faille and Heintzé [10] proposed a linearized Riemann solver which may be interpreted as a simplified version of the approach of Romate. However, their suggested scheme does not satisfy the Roe conditions, with the consequence that the numerical fluxes are generally discontinuous if there is a change of sign in an eigenvalue between neighbouring cells.

A more formal approach was undertaken by Toumi and Caruge [30] for a related model involving a mixture mass equation and a mixture energy equation. Based on a splitting of the flux into a "mixture" and "drift" part, they described how a Roe matrix could be obtained using the parameter-vector approach of Roe [22]. Unfortunately, the success of this approach relies heavily on the simplicity of the flux Jacobian of their model. Furthermore, their framework leads to integrals over the closure laws for which closed-form solutions do not generally exist.

Baudin et al. [2,3] suggested a relaxation scheme of the type proposed by Jin and Xin [14]. This is somewhat related to the Roe scheme in that one needs only to solve a linear Riemann problem at each cell interface. However, the relaxation parameters must be chosen with care to avoid excessive numerical dissipation.

In this paper, we propose an alternative method for constructing a Roe solver for the drift-flux model. To as large a degree as possible, we construct our Roe solver based on analytically derived averages. By this, we address computational complexity issues associated with previously derived Roe solvers. Furthermore, we are able to isolate the effect of the closure laws on the mathematical structure of the Roe linearization. Hence our analytically derived Roe matrix can be used in conjunction with arbitrary formulations of the closure laws. Nevertheless, special consideration will be given to the Zuber-Findlay law. 
The paper is organized as follows: In Section 1, we describe the two-phase flow model that we will be working with. In Section 1.1.3, we discuss the hydrodynamic closure law. In Section 1.2, we derive an analytical expression for the flux Jacobian of the model. In Section 2, we discuss various strategies for analytically constructing a Roe solver for systems of conservation laws. In Section 2.2, we adapt these strategies to the drift-flux model supplied with general closure laws. In Section 2.3, we present a method for obtaining a fully analytical Roe matrix for the special case of the Zuber-Findlay closure law. In Section 2.4, the main results of Section 2 are summarized. The numerical algorithm is described in Section 3.

In Section 4 we present numerical simulations, demonstrating accuracy and robustness properties of the scheme. Finally, our results are summarized in Section 5.

\section{THE DRIFT-FLUX MODEL}

\subsection{Model formulation}

The model that we will be concerned with may be written in the following vector form

$$
\frac{\partial \boldsymbol{q}}{\partial t}+\frac{\partial \boldsymbol{f}(\boldsymbol{q})}{\partial x}=\boldsymbol{s}(\boldsymbol{q}),
$$

where $\boldsymbol{q}$ is the vector of conserved variables, $\boldsymbol{f}$ is the vector of fluxes, and $\boldsymbol{s}(\boldsymbol{q})$ is the vector of sources. They are given by

$$
\begin{aligned}
& \boldsymbol{q}=\left[\begin{array}{c}
\rho_{\mathrm{g}} \alpha_{\mathrm{g}} \\
\rho_{\ell} \alpha_{\ell} \\
\rho_{\mathrm{g}} \alpha_{\mathrm{g}} v_{\mathrm{g}}+\rho_{\ell} \alpha_{\ell} v_{\ell}
\end{array}\right]=\left[\begin{array}{c}
m_{\mathrm{g}} \\
m_{\ell} \\
I_{\mathrm{g}}+I_{\ell}
\end{array}\right], \\
& \boldsymbol{f}(\boldsymbol{q})=\left[\begin{array}{c}
\rho_{\mathrm{g}} \alpha_{\mathrm{g}} v_{\mathrm{g}} \\
\rho_{\ell} \alpha_{\ell} v_{\ell} \\
\rho_{\mathrm{g}} \alpha_{\mathrm{g}} v_{\mathrm{g}}^{2}+\rho_{\ell} \alpha_{\ell} v_{\ell}^{2}+p
\end{array}\right]=\left[\begin{array}{c}
I_{\mathrm{g}} \\
I_{\ell} \\
I_{\mathrm{g}} v_{\mathrm{g}}+I_{\ell} v_{\ell}+p
\end{array}\right]
\end{aligned}
$$

and

$$
\boldsymbol{s}(\boldsymbol{q})=\left[\begin{array}{c}
0 \\
0 \\
-F_{\mathrm{w}}
\end{array}\right] .
$$

\subsubsection{Nomenclature}

In the following, we use the index $k \in[\mathrm{g}, \ell]$ to denote either the gas $(\mathrm{g})$ or liquid $(\ell)$ phase. For each phase, the variables are defined as follows:

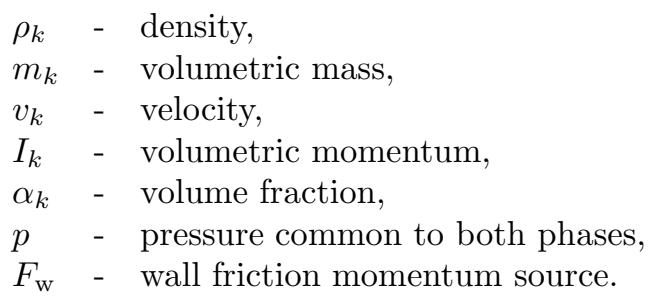

In the numerical examples presented in this paper, $F_{\mathrm{w}}$ is set equal to zero unless otherwise stated.

The volume fractions satisfy

$$
\alpha_{\mathrm{g}}+\alpha_{\ell}=1 .
$$

Dynamic mass and energy transfers are neglected; we consider isentropic or isothermal flows. In particular, this means that the pressure may be obtained as

$$
p=p\left(\rho_{\mathrm{g}}\right)=p\left(\rho_{\ell}\right) .
$$




\subsubsection{Thermodynamic submodels}

For the numerical simulations presented in this work, we assume that both the gas and liquid phases are compressible, where $p\left(\rho_{k}\right)$ are strictly increasing functions, i.e.

$$
\frac{\partial p}{\partial \rho_{k}}>0
$$

In particular, this implies that $p\left(\rho_{k}\right)$ are invertible functions, so that the densities may be expressed as

$$
\rho_{k}=\rho_{k}(p)
$$

In this work we consider locally linearized thermodynamic relations

$$
\rho_{\ell}=\rho_{\ell, 0}+\frac{p-p_{\ell, 0}}{c_{\ell}^{2}}
$$

and

where

$$
\rho_{\mathrm{g}}=\rho_{\mathrm{g}, 0}+\frac{p-p_{\mathrm{g}, 0}}{c_{\mathrm{g}}^{2}}
$$

and

$$
p_{k, 0}=p\left(\rho_{k, 0}\right)
$$

$$
c_{k}^{2} \equiv \frac{\partial p}{\partial \rho_{k}}\left(p_{k, 0}\right)
$$

Note that (10) and (11) can be written in the more condensed form

$$
p_{k}=c_{k}^{2}\left(\rho_{k}-\rho_{k}^{\circ}\right)
$$

where the parameter $\rho_{k}^{\circ}$ is given by

$$
\rho_{k}^{\circ}=\rho_{k, 0}-\frac{p_{k, 0}}{c_{k}^{2}}
$$

The values of the parameters $\rho_{k}^{\circ}$ and $c_{k}$ will be specified in Section 4 .

\subsubsection{Hydrodynamic submodels}

By far the most important aspect of the model is the hydrodynamic closure law, which is commonly expressed in the following general form

$$
v_{\mathrm{g}}-v_{\ell}=\Phi\left(m_{\mathrm{g}}, m_{\ell}, v_{\mathrm{g}}\right) .
$$

Provided that $\Phi$ is properly chosen for the application, Masella et al. [17] argue that the drift-flux model has a very general validity for practical two-phase flow problems.

The formulation of this law has a large effect on the flux Jacobian of the drift-flux model, and hence on the construction of the linearized Roe solver. A general approach for handling this difficulty is described in Section 2.2.7, where we explicitly express the Roe matrix as a function of $\Phi$. Hence our proposed Roe solver can be used in conjunction with arbitrary closure laws in the form (15).

Nevertheless, a special case of significant interest is the Zuber-Findlay [33] relation

$$
v_{\mathrm{g}}=K\left(\alpha_{\mathrm{g}} v_{\mathrm{g}}+\alpha_{\ell} v_{\ell}\right)+S
$$

where $K$ and $S$ are flow-dependent parameters. The validity of (16) is limited to the slug and bubbly flow regimes. However, for these regimes the Zuber-Findlay law has been experimentally verified for a broad range of parameters [4,12]. In the numerical Section 4, we put particular emphasis on the Zuber-Findlay law. A Roe linearization specially adapted to this law is presented in Section 2.3. 


\subsection{The Jacobian matrix}

An alternative formulation of the system (2) is the quasi-linear form

$$
\frac{\partial \boldsymbol{q}}{\partial t}+\boldsymbol{A}(\boldsymbol{q}) \frac{\partial \boldsymbol{q}}{\partial x}=s(\boldsymbol{q})
$$

where the flux Jacobian $\boldsymbol{A}(\boldsymbol{q})$ is defined as

$$
\boldsymbol{A}(\boldsymbol{q}) \equiv \frac{\partial \boldsymbol{f}}{\partial \boldsymbol{q}}=\left[\frac{\partial f_{i}}{\partial q_{j}}\right]
$$

In the following, we will derive an expression for $\boldsymbol{A}$. Towards this aim, we will follow the common practice of thermodynamics and take

$$
\left(\frac{\partial X}{\partial Y}\right)_{a, b}
$$

to mean the partial derivative of $X$ with respect to $Y$ under the assumption of constant $a$ and $b$.

\subsubsection{Some definitions}

We now define the following basic abbreviations:

$$
\begin{aligned}
\mu_{\mathrm{g}} & =\left(\frac{\partial \Phi}{\partial m_{\mathrm{g}}}\right)_{m_{\ell}, v_{\mathrm{g}}} \\
\mu_{\ell} & =\left(\frac{\partial \Phi}{\partial m_{\ell}}\right)_{m_{\mathrm{g}}, v_{\mathrm{g}}} \\
\mu_{\mathrm{v}} & =\left(\frac{\partial \Phi}{\partial v_{\mathrm{g}}}\right)_{m_{\mathrm{g}}, m_{\ell}} \\
\zeta & =\left(\frac{\partial v_{\ell}}{\partial v_{\mathrm{g}}}\right)_{m_{\mathrm{g}}, m_{\ell}} .
\end{aligned}
$$

We further define the pseudo mass $\varrho$ as

$$
\varrho=m_{\mathrm{g}}+\zeta m_{\ell} .
$$

Remark 1. We observe that by writing (1) as

$$
\mathrm{d} \Phi=\mathrm{d} v_{\mathrm{g}}-\mathrm{d} v_{\ell}
$$

we obtain from (22) and (23) the basic relation

$$
\mu_{\mathrm{v}}=1-\zeta
$$

We may now state a number of useful preliminary results:

Lemma 1. The gas velocity differential $\mathrm{d} v_{\mathrm{g}}$ may be written as

$$
\mathrm{d} v_{\mathrm{g}}=\frac{1}{\varrho}\left(\left(m_{\ell} \mu_{\mathrm{g}}-v_{\mathrm{g}}\right) \mathrm{d} q_{1}+\left(m_{\ell} \mu_{\ell}-v_{\ell}\right) \mathrm{d} q_{2}+\mathrm{d} q_{3}\right) .
$$

Proof. We may expand $\mathrm{d} q_{3}$ as

$$
\mathrm{d} q_{3}=m_{\mathrm{g}} \mathrm{d} v_{\mathrm{g}}+v_{\mathrm{g}} \mathrm{d} m_{\mathrm{g}}+v_{\ell} \mathrm{d} m_{\ell}+m_{\ell} \mathrm{d} v_{\ell} .
$$


Furthermore, the definitions (20)-(22) may be written in the form

$$
\mathrm{d} \Phi=\mu_{\mathrm{g}} \mathrm{d} m_{\mathrm{g}}+\mu_{\ell} \mathrm{d} m_{\ell}+\mu_{\mathrm{v}} \mathrm{d} v_{\mathrm{g}} .
$$

Using (26), we obtain from (25) and (29)

$$
\mathrm{d} v_{\ell}=\mathrm{d} v_{\mathrm{g}}-\mathrm{d} \Phi=\zeta \mathrm{d} v_{\mathrm{g}}-\mu_{\mathrm{g}} \mathrm{d} m_{\mathrm{g}}-\mu_{\ell} \mathrm{d} m_{\ell} .
$$

The result follows from substituting (30) into (28) and solving for $\mathrm{d} v_{\mathrm{g}}$.

Lemma 2. The liquid velocity differential $\mathrm{d} v_{\ell}$ may be written as

$$
\mathrm{d} v_{\ell}=\frac{1}{\varrho}\left(-\left(m_{\mathrm{g}} \mu_{\mathrm{g}}+\zeta v_{\mathrm{g}}\right) \mathrm{d} q_{1}-\left(m_{\mathrm{g}} \mu_{\ell}+\zeta v_{\ell}\right) \mathrm{d} q_{2}+\zeta \mathrm{d} q_{3}\right) .
$$

Proof. The result follows from substituting (27) into (30).

Lemma 3. The gas momentum differential $\mathrm{d} I_{\mathrm{g}}$ may be written as

$$
\mathrm{d} I_{\mathrm{g}}=\frac{1}{\varrho}\left(\left(m_{\mathrm{g}} m_{\ell} \mu_{\mathrm{g}}+\zeta m_{\ell} v_{\mathrm{g}}\right) \mathrm{d} q_{1}+\left(m_{\mathrm{g}} m_{\ell} \mu_{\ell}-m_{\mathrm{g}} v_{\ell}\right) \mathrm{d} q_{2}+m_{\mathrm{g}} \mathrm{d} q_{3}\right) .
$$

Proof. We may expand $\mathrm{d} I_{\mathrm{g}}$ as

$$
\mathrm{d} I_{\mathrm{g}}=m_{\mathrm{g}} \mathrm{d} v_{\mathrm{g}}+v_{\mathrm{g}} \mathrm{d} m_{\mathrm{g}}=m_{\mathrm{g}} \mathrm{d} v_{\mathrm{g}}+v_{\mathrm{g}} \mathrm{d} q_{1} .
$$

The result follows from substituting (27) into (33).

Lemma 4. The liquid momentum differential $\mathrm{d} I_{\ell}$ may be written as

$$
\mathrm{d} I_{\ell}=\frac{1}{\varrho}\left(-\left(m_{\mathrm{g}} m_{\ell} \mu_{\mathrm{g}}+\zeta m_{\ell} v_{\mathrm{g}}\right) \mathrm{d} q_{1}-\left(m_{\mathrm{g}} m_{\ell} \mu_{\ell}-m_{\mathrm{g}} v_{\ell}\right) \mathrm{d} q_{2}+\zeta m_{\ell} \mathrm{d} q_{3}\right) .
$$

Proof. Using the definition

it follows that

$$
\mathrm{d} q_{3}=\mathrm{d} I_{\mathrm{g}}+\mathrm{d} I_{\ell}
$$

The result follows from substituting (32) into (36).

$$
\mathrm{d} I_{\ell}=\mathrm{d} q_{3}-\mathrm{d} I_{\mathrm{g}}
$$

Lemma 5. The pressure differential $\mathrm{d} p$ may be written as

$$
\mathrm{d} p=\kappa\left(\rho_{\ell} \mathrm{d} q_{1}+\rho_{\mathrm{g}} \mathrm{d} q_{2}\right),
$$

where

$$
\kappa=\frac{1}{\left(\partial \rho_{\mathrm{g}} / \partial p\right) \rho_{\ell} \alpha_{\mathrm{g}}+\left(\partial \rho_{\ell} / \partial p\right) \rho_{\mathrm{g}} \alpha_{\ell}} .
$$

Proof. By the definitions in Section 1.1.1, we may rewrite $\alpha_{\mathrm{g}}+\alpha_{\ell}=1$ as

$$
\frac{m_{\mathrm{g}}}{\rho_{\mathrm{g}}(p)}+\frac{m_{\ell}}{\rho_{\ell}(p)}=1
$$

Differentiating (39) we obtain

$$
\frac{1}{\rho_{\mathrm{g}}} \mathrm{d} m_{\mathrm{g}}-\frac{m_{\mathrm{g}}}{\rho_{\mathrm{g}}^{2}} \frac{\partial \rho_{\mathrm{g}}}{\partial p} \mathrm{~d} p+\frac{1}{\rho_{\ell}} \mathrm{d} m_{\ell}-\frac{m_{\ell}}{\rho_{\ell}^{2}} \frac{\partial \rho_{\ell}}{\partial p} \mathrm{~d} p=0 .
$$


Solving for $\mathrm{d} p$ yields the desired result.

Lemma 6. The gas momentum convection differential $\mathrm{d}\left(I_{\mathrm{g}} v_{\mathrm{g}}\right)$ may be written as

$$
\begin{aligned}
\mathrm{d}\left(I_{\mathrm{g}} v_{\mathrm{g}}\right)= & \frac{1}{\varrho}\left(\left(2 m_{\mathrm{g}} m_{\ell} v_{\mathrm{g}} \mu_{\mathrm{g}}+\left(\zeta m_{\ell}-m_{\mathrm{g}}\right) v_{\mathrm{g}}^{2}\right) \mathrm{d} q_{1}\right. \\
& \left.+\left(2 m_{\mathrm{g}} m_{\ell} v_{\mathrm{g}} \mu_{\ell}-2 m_{\mathrm{g}} v_{\mathrm{g}} v_{\ell}\right) \mathrm{d} q_{2}+2 m_{\mathrm{g}} v_{\mathrm{g}} \mathrm{d} q_{3}\right)
\end{aligned}
$$

Proof. We may expand $\mathrm{d}\left(I_{\mathrm{g}} v_{\mathrm{g}}\right)$ as

$$
\mathrm{d}\left(I_{\mathrm{g}} v_{\mathrm{g}}\right)=I_{\mathrm{g}} \mathrm{d} v_{\mathrm{g}}+v_{\mathrm{g}} \mathrm{d} I_{\mathrm{g}}
$$

The result follows from substituting (27) and (32) into (42).

Lemma 7. The liquid momentum convection differential $\mathrm{d}\left(I_{\ell} v_{\ell}\right)$ may be written as

$$
\begin{aligned}
\mathrm{d}\left(I_{\ell} v_{\ell}\right)= & \frac{1}{\varrho}\left(-\left(2 m_{\mathrm{g}} m_{\ell} v_{\ell} \mu_{\mathrm{g}}+2 \zeta m_{\ell} v_{\mathrm{g}} v_{\ell}\right) \mathrm{d} q_{1}\right. \\
& \left.-\left(2 m_{\mathrm{g}} m_{\ell} v_{\ell} \mu_{\ell}+\left(\zeta m_{\ell}-m_{\mathrm{g}}\right) v_{\ell}^{2}\right) \mathrm{d} q_{2}+2 \zeta m_{\ell} v_{\ell} \mathrm{d} q_{3}\right) .
\end{aligned}
$$

Proof. We may expand $\mathrm{d}\left(I_{\ell} v_{\ell}\right)$ as

$$
\mathrm{d}\left(I_{\ell} v_{\ell}\right)=I_{\ell} \mathrm{d} v_{\ell}+v_{\ell} \mathrm{d} I_{\ell}
$$

The result follows from substituting (31) and (34) into (44).

Using these lemmas, we see that the Jacobian matrix can be written as

$$
\boldsymbol{A}(\boldsymbol{q})=\frac{1}{\varrho}\left[\begin{array}{ccc}
m_{\mathrm{g}} m_{\ell} \mu_{\mathrm{g}}+\zeta m_{\ell} v_{\mathrm{g}} & m_{\mathrm{g}} m_{\ell} \mu_{\ell}-m_{\mathrm{g}} v_{\ell} & m_{\mathrm{g}} \\
-\left(m_{\mathrm{g}} m_{\ell} \mu_{\mathrm{g}}+\zeta m_{\ell} v_{\mathrm{g}}\right) & m_{\mathrm{g}} v_{\ell}-m_{\mathrm{g}} m_{\ell} \mu_{\ell} & \zeta m_{\ell} \\
a_{31} & a_{32} & 2\left(m_{\mathrm{g}} v_{\mathrm{g}}+\zeta m_{\ell} v_{\ell}\right)
\end{array}\right]
$$

where

$$
a_{31}=\kappa \varrho \rho_{\ell}+2 m_{\mathrm{g}} m_{\ell} \mu_{\mathrm{g}}\left(v_{\mathrm{g}}-v_{\ell}\right)+\left(\zeta m_{\ell}-m_{\mathrm{g}}\right) v_{\mathrm{g}}^{2}-2 \zeta m_{\ell} v_{\mathrm{g}} v_{\ell}
$$

and

$$
a_{32}=\kappa \varrho \rho_{\mathrm{g}}+2 m_{\mathrm{g}} m_{\ell} \mu_{\ell}\left(v_{\mathrm{g}}-v_{\ell}\right)-\left(\zeta m_{\ell}-m_{\mathrm{g}}\right) v_{\ell}^{2}-2 m_{\mathrm{g}} v_{\mathrm{g}} v_{\ell} .
$$

\section{The Roe linearization}

The essence of Roe's method [22] is the replacement of the original nonlinear problem

$$
\frac{\partial \boldsymbol{q}}{\partial t}+\frac{\partial}{\partial x} \boldsymbol{f}(\boldsymbol{q})=\mathbf{0}
$$

by a linearized problem defined locally at each cell interface;

$$
\frac{\partial \hat{\boldsymbol{q}}}{\partial t}+\hat{\boldsymbol{A}}_{i-1 / 2} \frac{\partial \hat{\boldsymbol{q}}}{\partial x}=\mathbf{0}
$$

In the context of Roe's method, the matrix $\hat{\boldsymbol{A}}_{i-1 / 2}$ is expressed as a function of the left and right states as $\hat{\boldsymbol{A}}\left(\boldsymbol{q}^{\mathrm{L}}, \boldsymbol{q}^{\mathrm{R}}\right)$, and must satisfy the following conditions:

$\mathrm{R} 1: \hat{\boldsymbol{A}}\left(\boldsymbol{q}^{\mathrm{L}}, \boldsymbol{q}^{\mathrm{R}}\right)\left(\boldsymbol{q}^{\mathrm{R}}-\boldsymbol{q}^{\mathrm{L}}\right)=\boldsymbol{f}\left(\boldsymbol{q}^{\mathrm{R}}\right)-\boldsymbol{f}\left(\boldsymbol{q}^{\mathrm{L}}\right) ;$

R2: $\hat{\boldsymbol{A}}\left(\boldsymbol{q}^{\mathrm{L}}, \boldsymbol{q}^{\mathrm{R}}\right)$ is diagonalizable with real eigenvalues;

$\mathrm{R} 3: \hat{\boldsymbol{A}}\left(\boldsymbol{q}^{\mathrm{L}}, \boldsymbol{q}^{\mathrm{R}}\right) \rightarrow \boldsymbol{A}(\boldsymbol{q})$ smoothly as $\boldsymbol{q}^{\mathrm{L}}, \boldsymbol{q}^{\mathrm{R}} \rightarrow \boldsymbol{q}$. 
The main difficulty with the construction of such a matrix $\hat{A}$ is commonly associated with the condition R1. However, here the condition R2 is also non-trivial as the drift-flux model we consider is itself only conditionally hyperbolic [5]. In particular, if the relative velocity $\Phi$ between the phases becomes too large, the mixture sound velocity becomes imaginary and the system takes on an elliptic nature, see Appendix A.

In particular, this means that the following negative result holds:

Proposition 1. For the general drift-flux model, no consistent Roe linearization can unconditionally satisfy R2.

Proof. Assume a Roe linearization satisfying R3. Assume further that $\boldsymbol{q}=\boldsymbol{q}^{\mathrm{L}}=\boldsymbol{q}^{\mathrm{R}}$ is in the non-hyperbolic region of the model. Then by $\mathrm{R} 3$ we obtain:

$$
\hat{\boldsymbol{A}}\left(\boldsymbol{q}^{\mathrm{L}}, \boldsymbol{q}^{\mathrm{R}}\right)=\boldsymbol{A}(\boldsymbol{q})
$$

and $\mathrm{R} 2$ is violated.

Hence in this paper, we will be content with deriving a scheme that only conditionally satisfies R2. Note that if $\boldsymbol{q}^{\mathrm{L}}$ and $\boldsymbol{q}^{\mathrm{R}}$ are sufficiently close and in the hyperbolic region of the model, R2 is more or less automatically guaranteed by R3.

In the following, we will consequently focus on proving that our Roe scheme satisfies the conditions R1 and R3. Although the complexity of the model precludes us from deriving exact hyperbolicity conditions, we will verify that $\mathrm{R} 2$ holds for the numerical simulations.

The hyperbolicity condititions are discussed in more detail in Appendix A.

\subsection{Linearization strategies}

In the event that the flux function $\boldsymbol{f}$ is a rational function of the components of $\boldsymbol{q}$, Roe [22] discusses two strategies to meet the condition R1:

Strategy 1 (direct algebraic manipulation). The following are discrete variants of the differential rules for rational functions:

$$
\begin{aligned}
\Delta(p \pm q) & =\Delta p \pm \Delta q, \\
\Delta(p q) & =\bar{p} \Delta q+\bar{q} \Delta p, \\
\Delta(1 / q) & =-\Delta q / \tilde{q}^{2},
\end{aligned}
$$

where $(\cdot)$ denotes an arithmetic and $(\tilde{*})$ denotes a geometric mean value. As any rational function $y\left(x_{1}, \ldots, x_{n}\right)$ can be constructed by a sequence of additions, multiplications and divisions on its arguments, it follows from (51)-(53) that any jump $\Delta y$ can generally be written in terms of jumps in $x_{r}$ as

$$
\Delta y\left(x_{1}, \ldots, x_{n}\right)=\sum_{r=1}^{n} k_{r} \Delta x_{r}
$$

where the coefficients $k_{r}$ are obtained by repeated application of (51)-(53) to $\Delta y$.

Now (54) may be extended to the flux vector $\boldsymbol{f}(\boldsymbol{q})$ to yield

$$
f_{i}\left(\boldsymbol{q}^{\mathrm{R}}\right)-f_{i}\left(\boldsymbol{q}^{\mathrm{L}}\right)=\sum_{j} \hat{A}_{i j}\left(q_{j}^{\mathrm{R}}-q_{j}^{\mathrm{L}}\right)
$$

where $\hat{A}_{i j}$, constructed by repeated application of (51)-(53), are the entries of $\hat{\boldsymbol{A}}$. 
Strategy $\mathbf{2}$ (parameter vectors). We assume that $\boldsymbol{f}$ and $\boldsymbol{q}$ may be expressed through a change of variables as

$$
\begin{aligned}
& \boldsymbol{f}=\boldsymbol{f}(\boldsymbol{w}) \\
& \boldsymbol{q}=\boldsymbol{q}(\boldsymbol{w}),
\end{aligned}
$$

for some parameter vector $\boldsymbol{w}(\boldsymbol{q})$, where the components of $\boldsymbol{f}$ are at most quadratic polynomials in the components of $\boldsymbol{w}$. Then, by (51) and (52), any jump in $\boldsymbol{f}$ is related to jumps in $\boldsymbol{w}$ exclusively through arithmetic averages, and the Roe matrix may be obtained as

$$
\hat{\boldsymbol{A}}\left(\boldsymbol{q}^{\mathrm{L}}, \boldsymbol{q}^{\mathrm{R}}\right)=\boldsymbol{A}\left(q\left(\frac{1}{2}\left(\boldsymbol{w}^{\mathrm{L}}+\boldsymbol{w}^{\mathrm{R}}\right)\right) .\right.
$$

Roe [22] presented a successful application of the parameter-vector approach to the 3-dimensional Euler equations. He also made the observation that several other systems of conservation laws possess a sufficiently simple structure allowing for a generalization of the approach.

Strategies 1 and 2 are both based on the assumption that the flux vector is a rational function of the conserved variables. For the drift-flux model, this may hold for simple thermodynamics and the Zuber-Findlay slip relation, but will not be the case for more general closure laws.

Consequently, we would here like to draw attention to the fact that for the most general case, an alternative approach exists where the Roe matrix may be expressed as a function of $\boldsymbol{f}$ and $\boldsymbol{q}$ directly. This may be achieved by replacing the Jacobian by suitable numerical flux derivatives, as described below.

Strategy 3 (flux differences). Assuming that $\boldsymbol{q}$ is an $N$-vector, we may write the flux function $\boldsymbol{f}$ as

$$
\boldsymbol{f}(\boldsymbol{q})=\boldsymbol{f}\left(q_{1}, q_{2}, \ldots, q_{N}\right) .
$$

We now introduce the p-component flux difference symbol $\Delta_{(p)}$, defined by

$$
\Delta_{(p)} \boldsymbol{f}\left(\boldsymbol{q}^{\mathrm{L}}, \boldsymbol{q}^{\mathrm{R}}\right)=\boldsymbol{f}\left(q_{1}^{\mathrm{R}}, \ldots, q_{p}^{\mathrm{R}}, q_{p+1}^{\mathrm{L}}, \ldots, q_{N}^{\mathrm{L}}\right)-\boldsymbol{f}\left(q_{1}^{\mathrm{R}}, \ldots, q_{p-1}^{\mathrm{R}}, q_{p}^{\mathrm{L}}, \ldots, q_{N}^{\mathrm{L}}\right),
$$

for left and right states $\boldsymbol{q}^{\mathrm{L}}$ and $\boldsymbol{q}^{\mathrm{R}}$, where $p \in[1, \ldots, N]$.

We may now state the following theorem:

Theorem 1. The $N \times N$ matrix $\hat{\boldsymbol{A}}$ given by

$$
\hat{\boldsymbol{A}}\left(\boldsymbol{q}^{\mathrm{L}}, \boldsymbol{q}^{\mathrm{R}}\right)=\left[\hat{A}_{i j}\right],
$$

where

$$
\hat{A}_{i j}= \begin{cases}\frac{\Delta_{(j)} f_{i}\left(\boldsymbol{q}^{\mathrm{L}}, \boldsymbol{q}^{\mathrm{R}}\right)}{q_{j}^{\mathrm{R}}-q_{j}^{\mathrm{L}}} & \text { for } q_{j}^{\mathrm{L}} \neq q_{j}^{\mathrm{R}} \\ \frac{\partial f_{i}}{\partial q_{j}}\left(q_{1}^{\mathrm{R}}, \ldots, q_{j-1}^{\mathrm{R}}, q_{j}^{\mathrm{L}}, \ldots, q_{N}^{\mathrm{L}}\right) & \text { otherwise, }\end{cases}
$$

satisfies the Roe conditions $R 1$ and $R 3$ for all sufficiently smooth functions $\boldsymbol{f}(\boldsymbol{q})$.

Proof. By (61)-(62) we obtain

$$
\left[\hat{\boldsymbol{A}}\left(\boldsymbol{q}^{\mathrm{R}}-\boldsymbol{q}^{\mathrm{L}}\right)\right]_{i}=\sum_{j} \Delta_{(j)} f_{i}\left(\boldsymbol{q}^{\mathrm{L}}, \boldsymbol{q}^{\mathrm{R}}\right) \quad \forall i
$$

By substituting (60) into (63), we may simplify (63) to

$$
\left[\hat{\boldsymbol{A}}\left(\boldsymbol{q}^{\mathrm{R}}-\boldsymbol{q}^{\mathrm{L}}\right)\right]_{i}=\boldsymbol{f}_{i}\left(\boldsymbol{q}^{\mathrm{R}}\right)-\boldsymbol{f}_{i}\left(\boldsymbol{q}^{\mathrm{L}}\right) \quad \forall i,
$$


by cancelling the terms of opposite sign. This is the requirement R1.

Furthermore, by writing

$$
\boldsymbol{q}^{\mathrm{R}}=\boldsymbol{q}^{\mathrm{L}}+\varepsilon
$$

it follows from the definition of the partial derivative that

$$
\lim _{\varepsilon \rightarrow 0} \hat{A}_{i j}=\frac{\partial f_{i}}{\partial q_{j}}
$$

which is the requirement R3.

Remark 2. Note that Strategy 3 by no means guarantees that R2 is satisfied, even if it is applied to an unconditionally hyperbolic model.

Remark 3. Strategy 3 has the advantage of not making any assumptions about the flux function. On the other hand, if Strategies 1 and 2 are applicable, they generally lead to computationally cheaper algorithms.

\subsection{Considerations for the drift-flux model}

As previously discussed, the formulations of the hydrodynamic and thermodynamic closure laws are in general not available as analytical expressions. This leads to the conclusion that the parameter-vector approach will not be fruitful for the drift-flux model in general.

Instead, we will base our approach on Strategies 1 and 3 above. In particular, we will use Strategy 1 to isolate the irrational part of the flux function associated with the closure laws. In the most general case, a reduced version of Strategy 3 can then be applied to this irrational part.

In this paper, we wish to emphasize a somewhat unrecognized advantage associated with Strategy 1 - the large amount of freedom it presents us with in the construction of the Roe solver. In particular, we will take advantage of the following theorem:

Theorem 2. Let the flux vector $\boldsymbol{f}(\boldsymbol{q})$ be written as a sum of individual contributions

$$
\boldsymbol{f}(\boldsymbol{q})=\sum_{r} \boldsymbol{f}_{r}(\boldsymbol{q})
$$

Assume that with each $\boldsymbol{f}_{r}$, there is an associated matrix $\hat{\boldsymbol{A}}_{r}$ satisfying the Roe conditions $\mathrm{R} 1$ and $\mathrm{R} 3$ with respect to $\boldsymbol{f}_{r}$. Then the matrix

$$
\hat{\boldsymbol{A}}=\sum_{r} \hat{\boldsymbol{A}}_{r}
$$

satisfies both

(i) the Roe condition $\mathrm{R} 1$

(ii) the Roe condition $\mathrm{R} 3$

with respect to $\boldsymbol{f}(\boldsymbol{q})$.

Proof. From the Roe condition R1, (i) directly follows from linearity of matrix multiplication. Furthermore,

(ii) directly follows from the sum rule in differentiation.

\subsubsection{A flux-splitting strategy}

To derive our scheme, we apply the Roe condition R1 sequentially to the various parts of the equation system (2). In particular, by Theorem 2 we express the Roe matrix $\hat{A}$ as a sum of individual contributions

$$
\hat{\boldsymbol{A}}=\hat{\boldsymbol{A}}_{m}+\hat{\boldsymbol{A}}_{\mathrm{g}}+\hat{\boldsymbol{A}}_{\ell}+\hat{\boldsymbol{A}}_{p} .
$$


Hence, by not insisting that the Roe matrix must be written in the form (58), we are able to construct a valid matrix that

- consists almost entirely of simple arithmetic averages;

- allows the Roe-averaging of the closure laws to be isolated as fully independent problems.

We then demonstrate that Strategy 3 allows us to obtain a Roe-averaging of the closure laws with general validity. For the special case of the Zuber-Findlay closure law (16), an approach based on Strategy 1 allows us to directly obtain a fully analytical Roe matrix expressed in terms of the physical variables.

\subsubsection{Mass equations}

We first look for appropriate Roe averages for the mass conservation part of the system, i.e. we seek the submatrix

$$
\hat{\boldsymbol{A}}_{m}=\frac{1}{\hat{\varrho}}\left[\begin{array}{ccc}
\hat{m}_{\mathrm{g}} \hat{m}_{\ell} \hat{\mu}_{\mathrm{g}}+\hat{\zeta} \hat{m}_{\ell} \hat{v}_{\mathrm{g}} & \hat{m}_{\mathrm{g}} \hat{m}_{\ell} \hat{\mu}_{\ell}-\hat{m}_{\mathrm{g}} \hat{v}_{\ell} & \hat{m}_{\mathrm{g}} \\
-\left(\hat{m}_{\mathrm{g}} \hat{m}_{\ell} \hat{\mu}_{\mathrm{g}}+\hat{\zeta} \hat{m}_{\ell} \hat{v}_{\mathrm{g}}\right) & \hat{m}_{\mathrm{g}} \hat{v}_{\ell}-\hat{m}_{\mathrm{g}} \hat{m}_{\ell} \hat{\mu}_{\ell} & \hat{\zeta} \hat{m}_{\ell} \\
0 & 0 & 0
\end{array}\right]
$$

corresponding to the convective mass-flux vector

$$
\boldsymbol{f}_{m}(\boldsymbol{q})=\left[\begin{array}{c}
m_{\mathrm{g}} v_{\mathrm{g}} \\
m_{\ell} v_{\ell} \\
0
\end{array}\right]
$$

The Roe condition R1 yields two equations, which in vector form become

$$
\hat{\boldsymbol{A}}_{m}\left(\boldsymbol{q}^{\mathrm{R}}-\boldsymbol{q}^{\mathrm{L}}\right)=\boldsymbol{f}_{m}\left(\boldsymbol{q}^{\mathrm{R}}\right)-\boldsymbol{f}_{m}\left(\boldsymbol{q}^{\mathrm{L}}\right) .
$$

Following (24) and (26), we insist that

$$
\begin{aligned}
\hat{\varrho} & =\hat{m}_{\mathrm{g}}+\hat{\zeta} \hat{m}_{\ell} \\
\hat{\mu_{\mathrm{v}}} & =1-\hat{\zeta} .
\end{aligned}
$$

Given that the flux function $\boldsymbol{f}_{m}$ is quadratic in the variables $\left(m_{k}, v_{k}\right)$, the following averages are suggested by (52):

$$
\begin{aligned}
\hat{m}_{\mathrm{g}} & =\frac{1}{2}\left(m_{\mathrm{g}}^{\mathrm{L}}+m_{\mathrm{g}}^{\mathrm{R}}\right) \\
\hat{m}_{\ell} & =\frac{1}{2}\left(m_{\ell}^{\mathrm{L}}+m_{\ell}^{\mathrm{R}}\right) \\
\hat{v}_{\mathrm{g}} & =\frac{1}{2}\left(v_{\mathrm{g}}^{\mathrm{L}}+v_{\mathrm{g}}^{\mathrm{R}}\right) \\
\hat{v}_{\ell} & =\frac{1}{2}\left(v_{\ell}^{\mathrm{L}}+v_{\ell}^{\mathrm{R}}\right) .
\end{aligned}
$$

Substitution of (73)-(78) into (70) and (72) causes many terms to cancel out, and we are left with

$$
\hat{\mu_{\mathrm{g}}}\left(m_{\mathrm{g}}^{\mathrm{R}}-m_{\mathrm{g}}^{\mathrm{L}}\right)+\hat{\mu_{\ell}}\left(m_{\ell}^{\mathrm{R}}-m_{\ell}^{\mathrm{L}}\right)+\hat{\mu_{\mathrm{v}}}\left(v_{\mathrm{g}}^{\mathrm{R}}-v_{\mathrm{g}}^{\mathrm{L}}\right)=\Phi^{\mathrm{R}}-\Phi^{\mathrm{L}} .
$$

In other words, we have isolated the terms involving the closure law $\Phi$. Note that (79) is consistent with the definitions (20)-(22) in the sense that it is trivially satisfied in the case that $\left(\mu_{\mathrm{g}}, \mu_{\ell}, \mu_{\mathrm{v}}\right)$ are constant. In general, $\left(\mu_{\mathrm{g}}, \mu_{\ell}, \mu_{\mathrm{v}}\right)$ are not constant and we must derive suitable averages $\hat{\mu}$ satisfying the Roe-like condition (79). This is discussed in Sections 2.2.7 and 2.3. 


\subsubsection{Momentum convection}

We split the convective momentum flux as follows:

$$
\boldsymbol{f}_{I}(\boldsymbol{q})=\left[\begin{array}{c}
0 \\
0 \\
m_{\mathrm{g}} v_{\mathrm{g}}^{2}+m_{\ell} v_{\ell}^{2}
\end{array}\right]=\boldsymbol{f}_{\mathrm{g}}(\boldsymbol{q})+\boldsymbol{f}_{\ell}(\boldsymbol{q})
$$

where

and

$$
\boldsymbol{f}_{\mathrm{g}}(\boldsymbol{q})=\left[\begin{array}{c}
0 \\
0 \\
m_{\mathrm{g}} v_{\mathrm{g}}^{2}
\end{array}\right]
$$

$$
\boldsymbol{f}_{\ell}(\boldsymbol{q})=\left[\begin{array}{c}
0 \\
0 \\
m_{\ell} v_{\ell}^{2}
\end{array}\right]
$$

\subsubsection{Gas momentum convection}

We now seek Roe averages for the Jacobian submatrix

$$
\boldsymbol{A}_{\mathrm{g}}(\boldsymbol{q})=\frac{\partial \boldsymbol{f}_{\mathrm{g}}}{\partial \boldsymbol{q}}=\frac{1}{\varrho}\left[\begin{array}{ccc}
0 & 0 & 0 \\
0 & 0 & 0 \\
2 m_{\mathrm{g}} m_{\ell} v_{\mathrm{g}} \mu_{\mathrm{g}}+\left(\zeta m_{\ell}-m_{\mathrm{g}}\right) v_{\mathrm{g}}^{2} & 2 m_{\mathrm{g}} m_{\ell} v_{\mathrm{g}} \mu_{\ell}-2 m_{\mathrm{g}} v_{\mathrm{g}} v_{\ell} & 2 m_{\mathrm{g}} v_{\mathrm{g}}
\end{array}\right] .
$$

In particular, we observe that if we look for Roe averages of the form

$$
\begin{aligned}
& \hat{A}_{\mathrm{g}, 31}=\frac{1}{\hat{\varrho}}\left(2 \hat{m}_{\mathrm{g}} \hat{m}_{\ell}{\tilde{v_{\mathrm{g}}}}_{\hat{\mu_{\mathrm{g}}}}+2 \hat{\zeta} \hat{m}_{\ell} \hat{v}_{\mathrm{g}} \tilde{v}_{\mathrm{g}}-\left(\hat{\zeta} \hat{m}_{\ell}+\hat{m}_{\mathrm{g}}\right){\tilde{v_{\mathrm{g}}}}^{2}\right) \\
& \hat{A}_{\mathrm{g}, 32}=\frac{1}{\hat{\varrho}}\left(2 \hat{m}_{\mathrm{g}} \hat{m}_{\ell} \tilde{v}_{\mathrm{g}} \hat{\mu}_{\ell}-2 \hat{m}_{\mathrm{g}} \tilde{v}_{\mathrm{g}} \hat{v}_{\ell}\right) \\
& \hat{A}_{\mathrm{g}, 33}=\frac{1}{\hat{\varrho}}\left(2 \hat{m}_{\mathrm{g}}{\tilde{v_{\mathrm{g}}}}\right) \text {, }
\end{aligned}
$$

involving the assumption of two different Roe-averaged gas velocities $\tilde{v_{\mathrm{g}}}$ and $\hat{v_{\mathrm{g}}}$, we may write (84)-(86) as

$$
\begin{aligned}
& \hat{A}_{\mathrm{g}, 31}=2 \tilde{v_{\mathrm{g}}} \hat{A}_{m, 11}-{\tilde{v_{\mathrm{g}}}}^{2} \\
& \hat{A}_{\mathrm{g}, 32}=2 \tilde{v_{\mathrm{g}}} \hat{A}_{m, 12} \\
& \hat{A}_{\mathrm{g}, 33}=2 \tilde{v_{\mathrm{g}}} \hat{A}_{m, 13},
\end{aligned}
$$

where $\hat{\boldsymbol{A}}_{m}$ is the mass Roe matrix (70).

Remark 4. Note that $\hat{A}_{\mathrm{g}, 31}$ can equivalently be written as

$$
\left.\hat{A}_{\mathrm{g}, 31}=\frac{1}{\hat{\varrho}}\left(2 \hat{m_{\mathrm{g}}} \hat{m_{\ell}} \tilde{v_{\mathrm{g}}} \hat{\mu_{\mathrm{g}}}+\left(\hat{\zeta} \hat{m}_{\ell}-\hat{m}_{\mathrm{g}}\right){\tilde{\mathrm{g}_{\mathrm{g}}}}^{2}+2 \hat{\zeta} \hat{m}_{\ell}{\tilde{v_{\mathrm{g}}}}_{\left(\hat{v_{\mathrm{g}}}\right.}-\tilde{v_{\mathrm{g}}}\right)\right)
$$

where the last term vanishes when $\hat{v}_{\mathrm{g}}=\tilde{v}_{\mathrm{g}}$ (in accordance with the condition R3).

By the gas mass equation (72), the condition R1 on $\hat{\boldsymbol{A}}_{\mathrm{g}}$ simply becomes

$$
{\tilde{v_{\mathrm{g}}}}^{2}\left(m_{\mathrm{g}}^{\mathrm{R}}-m_{\mathrm{g}}^{\mathrm{L}}\right)-2 \tilde{v_{\mathrm{g}}}\left(m_{\mathrm{g}}^{\mathrm{R}} v_{\mathrm{g}}^{\mathrm{R}}-m_{\mathrm{g}}^{\mathrm{L}} v_{\mathrm{g}}^{\mathrm{L}}\right)+\left(m_{\mathrm{g}} v_{\mathrm{g}}^{2}\right)^{\mathrm{R}}-\left(m_{\mathrm{g}} v_{\mathrm{g}}^{2}\right)^{\mathrm{L}}=0,
$$


the solution of which is the standard Roe-averaged velocity, familiar from the Euler equations

$$
\tilde{v_{\mathrm{g}}}=\frac{\sqrt{m_{\mathrm{g}}^{\mathrm{L}}} v_{\mathrm{g}}^{\mathrm{L}}+\sqrt{m_{\mathrm{g}}^{\mathrm{R}}} v_{\mathrm{g}}^{\mathrm{R}}}{\sqrt{m_{\mathrm{g}}^{\mathrm{L}}}+\sqrt{m_{\mathrm{g}}^{\mathrm{R}}}}
$$

Here the gas mass $m_{\mathrm{g}}$ takes the place of the density $\rho$.

Hence a Roe average $\hat{\boldsymbol{A}}_{\mathrm{g}}$ for the gas momentum convection submatrix (83) is obtained rather nicely; the "hat" averages of $(84)-(86)$ are the simple arithmetic averages $(75)-(78)$, whereas the "tilde"-averaged gas velocity $\tilde{v}_{\mathrm{g}}$ of (84)-(86) is given by (92).

Remark 5. The simultaneous application of two different velocity averages $\tilde{v}_{\mathrm{g}}$ and $\hat{v}_{\mathrm{g}}$ here allows for a significantly simplified algebraic structure of the Roe matrix $\hat{\boldsymbol{A}}$.

\subsubsection{Liquid momentum convection}

We seek Roe averages for the Jacobian submatrix

$$
\boldsymbol{A}_{\ell}(\boldsymbol{q})=\frac{\partial \boldsymbol{f}_{\ell}}{\partial \boldsymbol{q}}=\frac{1}{\varrho}\left[\begin{array}{ccc}
0 & 0 & 0 \\
0 & 0 & 0 \\
-\left(2 m_{\mathrm{g}} m_{\ell} v_{\ell} \mu_{\mathrm{g}}+2 \zeta m_{\ell} v_{\mathrm{g}} v_{\ell}\right) & -\left(2 m_{\mathrm{g}} m_{\ell} v_{\ell} \mu_{\ell}+\left(\zeta m_{\ell}-m_{\mathrm{g}}\right) v_{\ell}^{2}\right) & 2 \zeta m_{\ell} v_{\ell}
\end{array}\right] .
$$

We proceed in a fully equivalent fashion as for the gas momentum convection, i.e. we look for Roe averages of the form

$$
\begin{aligned}
& \hat{A}_{\ell, 31}=-\frac{1}{\hat{\varrho}}\left(2 \hat{m}_{\mathrm{g}} \hat{m}_{\ell} \tilde{v}_{\ell} \hat{\mu}_{\mathrm{g}}+2 \hat{\zeta} \hat{m}_{\ell} \hat{v}_{\mathrm{g}} \tilde{v}_{\ell}\right) \\
& \hat{A}_{\ell, 32}=\frac{1}{\hat{\varrho}}\left(2 \hat{m}_{\mathrm{g}} \tilde{v}_{\ell} \hat{v}_{\ell}-2 \hat{m}_{\mathrm{g}} \hat{m}_{\ell} \tilde{v}_{\ell} \hat{\mu}_{\ell}-\left(\hat{m}_{\mathrm{g}}+\hat{\zeta} \hat{m}_{\ell}\right) \tilde{v}_{\ell}{ }^{2}\right) \\
& \hat{A}_{\ell, 33}=\frac{1}{\hat{\varrho}}\left(2 \hat{\zeta} \hat{m}_{\ell} \tilde{v}_{\ell}\right) .
\end{aligned}
$$

Remark 6. Note that $\hat{A}_{\ell, 32}$ can equivalently be written as

$$
\hat{A}_{\ell, 32}=\frac{1}{\hat{\varrho}}\left(-\left(2 \hat{m}_{\mathrm{g}} \hat{m}_{\ell} \tilde{v}_{\ell} \hat{\mu}_{\ell}+\left(\hat{\zeta} \hat{m}_{\ell}-\hat{m}_{\mathrm{g}}\right) \tilde{v}_{\ell}^{2}\right)+2 \hat{m}_{\mathrm{g}}{\tilde{v_{\ell}}}\left(\hat{v}_{\ell}-\tilde{v}_{\ell}\right)\right)
$$

where the last term vanishes when $\hat{v}_{\ell}=\tilde{v}_{\ell}$ (in accordance with the condition R3).

As for the gas momentum convection, we may express (94)-(96) in terms of the mass Roe matrix $\hat{\boldsymbol{A}}_{m}(70)$ as

$$
\begin{aligned}
\hat{A}_{\ell, 31} & =2 \tilde{v}_{\ell} \hat{A}_{m, 21} \\
\hat{A}_{\ell, 32} & =2 \tilde{v}_{\ell} \hat{A}_{m, 22}-\tilde{v}_{\ell}^{2} \\
\hat{A}_{\ell, 33} & =2 \tilde{v}_{\ell} \hat{A}_{m, 23} .
\end{aligned}
$$

By the liquid mass equation (72), the Roe momentum equation now reduces to

$$
{\tilde{v_{\ell}}}^{2}\left(m_{\ell}^{\mathrm{R}}-m_{\ell}^{\mathrm{L}}\right)-2 \tilde{v}_{\ell}\left(m_{\ell}^{\mathrm{R}} v_{\ell}^{\mathrm{R}}-m_{\ell}^{\mathrm{L}} v_{\ell}^{\mathrm{L}}\right)+\left(m_{\ell} v_{\ell}^{2}\right)^{\mathrm{R}}-\left(m_{\ell} v_{\ell}^{2}\right)^{\mathrm{L}}=0,
$$

with corresponding solution

$$
\tilde{v}_{\ell}=\frac{\sqrt{m_{\ell}^{\mathrm{L}}} v_{\ell}^{\mathrm{L}}+\sqrt{m_{\ell}^{\mathrm{R}}} v_{\ell}^{\mathrm{R}}}{\sqrt{m_{\ell}^{\mathrm{L}}}+\sqrt{m_{\ell}^{\mathrm{R}}}}
$$


In summary, a Roe average $\hat{\boldsymbol{A}}_{\ell}$ for the liquid momentum convection submatrix (93) is obtained as follows; the "hat" averages of (94)-(96) are the simple arithmetic averages (75)-(78), whereas the "tilde"-averaged liquid velocity $\tilde{v}_{\ell}$ of $(94)-(96)$ is given by (102).

\subsubsection{Pressure terms}

We here seek the Roe submatrix

$$
\hat{\boldsymbol{A}}_{p}=\left[\begin{array}{ccc}
0 & 0 & 0 \\
0 & 0 & 0 \\
\hat{\kappa} \hat{\rho}_{\ell} & \hat{\kappa} \hat{\rho}_{\mathrm{g}} & 0
\end{array}\right]
$$

corresponding to the flux vector

$$
\boldsymbol{f}_{p}(\boldsymbol{q})=\left[\begin{array}{l}
0 \\
0 \\
p
\end{array}\right]
$$

Writing (38) as

$$
\hat{\kappa}=\left(\widehat{\partial_{p} \rho_{\mathrm{g}}} \hat{\rho_{\ell}} \hat{\alpha_{\mathrm{g}}}+\widehat{\partial_{p} \rho_{\ell}} \hat{\rho_{\mathrm{g}}} \hat{\alpha_{\ell}}\right)^{-1}
$$

we obtain the equation

$$
\frac{\hat{\rho}_{\ell}\left(m_{\mathrm{g}}^{\mathrm{R}}-m_{\mathrm{g}}^{\mathrm{L}}\right)+\hat{\rho_{\mathrm{g}}}\left(m_{\ell}^{\mathrm{R}}-m_{\ell}^{\mathrm{L}}\right)}{\widehat{\partial_{p} \rho_{\mathrm{g}}} \hat{\rho_{\ell}} \hat{\alpha_{\mathrm{g}}}+\widehat{\partial_{p} \rho_{\ell}} \hat{\rho_{\mathrm{g}}} \hat{\alpha_{\ell}}}=p^{\mathrm{R}}-p^{\mathrm{L}}
$$

by the Roe condition R1.

The averages $\widehat{\partial_{p} \rho_{k}}$ indirectly involve the thermodynamic closure law, which, as previously discussed, may not be available in algebraic form. We hence suggest to apply Strategy 3 for the Roe-averaging of these terms. Taking advantage of the fact that we assume density models of the form (7), we suggest approximating these compressibility terms as

$$
\widehat{\partial_{p} \rho_{k}}= \begin{cases}\frac{\rho_{k}^{\mathrm{R}}-\rho_{k}^{\mathrm{L}}}{p^{\mathrm{R}}-p^{\mathrm{L}}} & \text { for } p^{\mathrm{L}} \neq p^{\mathrm{R}} \\ \left(\partial_{p} \rho_{k}\right)^{\mathrm{L}} & \text { otherwise. }\end{cases}
$$

Substituting (107) in (106) we obtain

$$
\hat{\rho_{\ell}}\left(m_{\mathrm{g}}^{\mathrm{R}}-m_{\mathrm{g}}^{\mathrm{L}}\right)+\hat{\rho_{\mathrm{g}}}\left(m_{\ell}^{\mathrm{R}}-m_{\ell}^{\mathrm{L}}\right)=\hat{\rho_{\mathrm{g}}} \hat{\alpha_{\ell}}\left(\rho_{\ell}^{\mathrm{R}}-\rho_{\ell}^{\mathrm{L}}\right)+\hat{\rho_{\ell}} \hat{\alpha_{\mathrm{g}}}\left(\rho_{\mathrm{g}}^{\mathrm{R}}-\rho_{\mathrm{g}}^{\mathrm{L}}\right),
$$

which is satisfied by the arithmetic averages

$$
\begin{aligned}
\hat{\alpha_{\ell}} & =\frac{1}{2}\left(\alpha_{\ell}^{\mathrm{L}}+\alpha_{\ell}^{\mathrm{R}}\right) \\
\hat{\alpha_{\mathrm{g}}} & =\frac{1}{2}\left(\alpha_{\mathrm{g}}^{\mathrm{L}}+\alpha_{\mathrm{g}}^{\mathrm{R}}\right) \\
\hat{\rho_{\mathrm{g}}} & =\frac{1}{2}\left(\rho_{\mathrm{g}}^{\mathrm{L}}+\rho_{\mathrm{g}}^{\mathrm{R}}\right) \\
\hat{\rho_{\ell}} & =\frac{1}{2}\left(\rho_{\ell}^{\mathrm{L}}+\rho_{\ell}^{\mathrm{R}}\right),
\end{aligned}
$$

where we have used that

$$
m_{k}=\rho_{k} \alpha_{k} .
$$




\subsubsection{The slip relation}

We now aim to obtain Roe averages $\left(\hat{\mu_{\mathrm{g}}}, \hat{\mu_{\ell}}, \hat{\mu_{\mathrm{v}}}\right)$ valid for general hydrodynamic closure laws $\Phi\left(m_{\mathrm{g}}, m_{\ell}, v_{\mathrm{g}}\right)$. Remark 3 consequently suggests that we should apply Strategy 3 to obtain these averages.

As noted in Section 2.2.2, the condition R1 dictates that the averages must satisfy

$$
\hat{\mu_{\mathrm{g}}}\left(m_{\mathrm{g}}^{\mathrm{R}}-m_{\mathrm{g}}^{\mathrm{L}}\right)+\hat{\mu}_{\ell}\left(m_{\ell}^{\mathrm{R}}-m_{\ell}^{\mathrm{L}}\right)+\hat{\mu_{\mathrm{v}}}\left(v_{\mathrm{g}}^{\mathrm{R}}-v_{\mathrm{g}}^{\mathrm{L}}\right)=\Phi^{\mathrm{R}}-\Phi^{\mathrm{L}} .
$$

Application of Strategy 3 directly yields

$$
\begin{aligned}
& \hat{\mu_{\mathrm{g}}}= \begin{cases}\frac{\Phi\left(m_{\mathrm{g}}^{\mathrm{R}}, m_{\ell}^{\mathrm{L}}, v_{\mathrm{g}}^{\mathrm{L}}\right)-\Phi\left(m_{\mathrm{g}}^{\mathrm{L}}, m_{\ell}^{\mathrm{L}}, v_{\mathrm{g}}^{\mathrm{L}}\right)}{m_{\mathrm{g}}^{\mathrm{R}}-m_{\mathrm{g}}^{\mathrm{L}}} & \text { for } m_{\mathrm{g}}^{\mathrm{L}} \neq m_{\mathrm{g}}^{\mathrm{R}} \\
\mu_{\mathrm{g}}\left(m_{\mathrm{g}}^{\mathrm{L}}, m_{\ell}^{\mathrm{L}}, v_{\mathrm{g}}^{\mathrm{L}}\right) & \text { otherwise }\end{cases} \\
& \hat{\mu_{\ell}}= \begin{cases}\frac{\Phi\left(m_{\mathrm{g}}^{\mathrm{R}}, m_{\ell}^{\mathrm{R}}, v_{\mathrm{g}}^{\mathrm{L}}\right)-\Phi\left(m_{\mathrm{g}}^{\mathrm{R}}, m_{\ell}^{\mathrm{L}}, v_{\mathrm{g}}^{\mathrm{L}}\right)}{m_{\ell}^{\mathrm{R}}-m_{\ell}^{\mathrm{L}}} & \text { for } m_{\ell}^{\mathrm{L}} \neq m_{\ell}^{\mathrm{R}} \\
\mu_{\ell}\left(m_{\mathrm{g}}^{\mathrm{R}}, m_{\ell}^{\mathrm{L}}, v_{\mathrm{g}}^{\mathrm{L}}\right) & \text { otherwise }\end{cases} \\
& \hat{\mu_{\mathrm{v}}}= \begin{cases}\frac{\Phi\left(m_{\mathrm{g}}^{\mathrm{R}}, m_{\ell}^{\mathrm{R}}, v_{\mathrm{g}}^{\mathrm{R}}\right)-\Phi\left(m_{\mathrm{g}}^{\mathrm{R}}, m_{\ell}^{\mathrm{R}}, v_{\mathrm{g}}^{\mathrm{L}}\right)}{v_{\mathrm{g}}^{\mathrm{R}}-v_{\mathrm{g}}^{\mathrm{L}}} & \text { for } v_{\mathrm{g}}^{\mathrm{L}} \neq v_{\mathrm{g}}^{\mathrm{R}} \\
\mu_{\mathrm{v}}\left(m_{\mathrm{g}}^{\mathrm{R}}, m_{\ell}^{\mathrm{R}}, v_{\mathrm{g}}^{\mathrm{L}}\right) & \text { otherwise. }\end{cases}
\end{aligned}
$$

\subsection{The Zuber-Findlay law}

Although the averages derived in Section 2.2.7 are valid for general formulations of the hydrodynamic closure law, they may not always be optimal in terms of computational efficiency, as noted in Remark 3.

In this section, we derive explicit averages for the special case of the Zuber-Findlay slip relation [33]

$$
v_{\mathrm{g}}=K\left(\alpha_{\mathrm{g}} v_{\mathrm{g}}+\alpha_{\ell} v_{\ell}\right)+S
$$

which may be equivalently expressed as

$$
\Phi=\frac{(K-1) v_{\mathrm{g}}+S}{K \alpha_{\ell}}
$$

The slip derivatives for this particular relation are found to be

$$
\begin{aligned}
\mu_{\mathrm{v}} & =\frac{K-1}{K \alpha_{\ell}} \\
\mu_{\mathrm{g}} & =\left(v_{\mathrm{g}}-v_{\ell}\right) \kappa \frac{\partial \rho_{\ell}}{\partial p} \\
\mu_{\ell} & =-\left(v_{\mathrm{g}}-v_{\ell}\right) \kappa \frac{\alpha_{\mathrm{g}}}{\alpha_{\ell}} \frac{\partial \rho_{\mathrm{g}}}{\partial p}
\end{aligned}
$$

Roe-averages for these slip derivatives are now found by applying Strategy 1 to the requirement (114), as described below.

\subsubsection{A splitting of the slip relation}

We first note that $\Phi$ can be written as

$$
\Phi=f\left(v_{\mathrm{g}}\right) \cdot g\left(\alpha_{\ell}\right),
$$

where

$$
f\left(v_{\mathrm{g}}\right)=(K-1) v_{\mathrm{g}}+S
$$


and

$$
g\left(\alpha_{\ell}\right)=\left(K \alpha_{\ell}\right)^{-1}
$$

By (52) and (123) we obtain

$$
\begin{aligned}
\Phi^{\mathrm{R}}-\Phi^{\mathrm{L}}= & \frac{1}{2}\left(f\left(v_{\mathrm{g}}^{\mathrm{L}}\right)+f\left(v_{\mathrm{g}}^{\mathrm{R}}\right)\right)\left(g\left(\alpha_{\ell}^{\mathrm{R}}\right)-g\left(\alpha_{\ell}^{\mathrm{L}}\right)\right) \\
& +\frac{1}{2}\left(g\left(\alpha_{\ell}^{\mathrm{L}}\right)+g\left(\alpha_{\ell}^{\mathrm{R}}\right)\right)\left(f\left(v_{\mathrm{g}}^{\mathrm{R}}\right)-f\left(v_{\mathrm{g}}^{\mathrm{L}}\right)\right),
\end{aligned}
$$

which suggests a natural splitting of (114) into two separate equations:

$$
\hat{\mu_{\mathrm{g}}}\left(m_{\mathrm{g}}^{\mathrm{R}}-m_{\mathrm{g}}^{\mathrm{L}}\right)+\hat{\mu}_{\ell}\left(m_{\ell}^{\mathrm{R}}-m_{\ell}^{\mathrm{L}}\right)=\frac{1}{2}\left(f\left(v_{\mathrm{g}}^{\mathrm{L}}\right)+f\left(v_{\mathrm{g}}^{\mathrm{R}}\right)\right)\left(g\left(\alpha_{\ell}^{\mathrm{R}}\right)-g\left(\alpha_{\ell}^{\mathrm{L}}\right)\right)
$$

and

$$
\hat{\mu_{\mathrm{v}}}\left(v_{\mathrm{g}}^{\mathrm{R}}-v_{\mathrm{g}}^{\mathrm{L}}\right)=\frac{1}{2}\left(g\left(\alpha_{\ell}^{\mathrm{L}}\right)+g\left(\alpha_{\ell}^{\mathrm{R}}\right)\right)\left(f\left(v_{\mathrm{g}}^{\mathrm{R}}\right)-f\left(v_{\mathrm{g}}^{\mathrm{L}}\right)\right) .
$$

\subsubsection{The velocity slip derivative}

We now express the Roe-average of (120) as

$$
\hat{\mu_{\mathrm{v}}}=\frac{K-1}{K \tilde{\alpha_{\ell}}} .
$$

By (124) and (125), (128) then becomes

$$
\frac{K-1}{K \tilde{\alpha_{\ell}}}=\frac{K-1}{2 K}\left(\frac{1}{\alpha_{\ell}^{\mathrm{L}}}+\frac{1}{\alpha_{\ell}^{\mathrm{R}}}\right),
$$

which yields $\tilde{\alpha}_{\ell}\left(\alpha_{\ell}^{\mathrm{L}}, \alpha_{\ell}^{\mathrm{R}}\right)$ as the harmonic mean:

$$
\tilde{\alpha_{\ell}}=2 \frac{\alpha_{\ell}^{\mathrm{L}} \alpha_{\ell}^{\mathrm{R}}}{\alpha_{\ell}^{\mathrm{L}}+\alpha_{\ell}^{\mathrm{R}}} .
$$

\subsubsection{The mass slip derivatives}

We write the Roe-averages of (121) and (122) as

$$
\hat{\mu_{\mathrm{g}}}=\hat{\Phi} \hat{\kappa} \widehat{\partial_{p} \rho_{\ell}}
$$

and

By writing

$$
\hat{\mu_{\ell}}=-\hat{\Phi} \hat{\kappa} \frac{\hat{\alpha_{\mathrm{g}}}}{\hat{\alpha_{\ell}}} \widehat{\partial_{p} \rho_{\mathrm{g}}} .
$$

$$
\hat{\Phi}=\frac{(K-1) \hat{v}_{\mathrm{g}}+S}{K \tilde{\alpha}_{\ell}},
$$

where

$$
\begin{aligned}
& \hat{v_{\mathrm{g}}}=\frac{1}{2}\left(v_{\mathrm{g}}^{\mathrm{L}}+v_{\mathrm{g}}^{\mathrm{R}}\right) \\
& \tilde{\alpha_{\ell}}=2 \frac{\alpha_{\ell}^{\mathrm{L}} \alpha_{\ell}^{\mathrm{R}}}{\alpha_{\ell}^{\mathrm{L}}+\alpha_{\ell}^{\mathrm{R}}},
\end{aligned}
$$


(127) can, by use of (53), be rewritten as

$$
-\hat{\Phi} \frac{\alpha_{\ell}^{\mathrm{R}}-\alpha_{\ell}^{\mathrm{L}}}{\hat{\alpha}_{\ell}}=\hat{\Phi} \hat{\kappa} \widehat{\partial_{p} \rho_{\ell}}\left(m_{\mathrm{g}}^{\mathrm{R}}-m_{\mathrm{g}}^{\mathrm{L}}\right)-\hat{\Phi} \hat{\kappa} \frac{\hat{\alpha_{\mathrm{g}}}}{\hat{\alpha}_{\ell}} \widehat{\partial_{p} \rho_{\mathrm{g}}}\left(m_{\ell}^{\mathrm{R}}-m_{\ell}^{\mathrm{L}}\right),
$$

where we define

$$
\hat{\alpha_{k}}=\frac{1}{2}\left(\alpha_{k}^{\mathrm{L}}+\alpha_{k}^{\mathrm{R}}\right) .
$$

We now observe that the averages $\hat{\kappa}$ and $\widehat{\partial_{p} \rho_{k}}$, obtained in Section 2.2.6, do in fact also satisfy (137); together with (134) they yield valid Roe averages (132) and (133).

\subsection{The Roe matrix}

The preceeding analysis of Sections 2.2-2.3 may be summed up by the following proposition:

Proposition 2. The matrix

$$
\hat{\boldsymbol{A}}\left(\boldsymbol{q}^{\mathrm{L}}, \boldsymbol{q}^{\mathrm{R}}\right)=\frac{1}{\hat{\varrho}}\left[\begin{array}{ccc}
\hat{m}_{\mathrm{g}} \hat{m}_{\ell} \hat{\mu}_{\mathrm{g}}+\hat{\zeta} \hat{m}_{\ell} \hat{v}_{\mathrm{g}} & \hat{m}_{\mathrm{g}} \hat{m}_{\ell} \hat{\mu}_{\ell}-\hat{m}_{\mathrm{g}} \hat{v}_{\ell} & \hat{m}_{\mathrm{g}} \\
-\left(\hat{m}_{\mathrm{g}} \hat{m}_{\ell} \hat{\mu}_{\mathrm{g}}+\hat{\zeta} \hat{m}_{\ell} \hat{v}_{\mathrm{g}}\right) & \hat{m}_{\mathrm{g}} \hat{v}_{\ell}-\hat{m}_{\mathrm{g}} \hat{m}_{\ell} \hat{\mu}_{\ell} & \hat{\zeta} \hat{m}_{\ell} \\
\hat{a}_{31} & \hat{a}_{32} & 2\left(\hat{m}_{\mathrm{g}} \tilde{v}_{\mathrm{g}}+\hat{\zeta} \hat{m}_{\ell} \tilde{v}_{\ell}\right)
\end{array}\right]
$$

where

$$
\begin{aligned}
& \hat{a}_{31}=\hat{\kappa} \hat{\varrho} \hat{\rho}_{\ell}+2 \hat{m}_{\mathrm{g}} \hat{m}_{\ell} \tilde{v_{\mathrm{g}}} \hat{\mu_{\mathrm{g}}}+2 \hat{\zeta} \hat{m}_{\ell} \hat{v_{\mathrm{g}}} \tilde{v_{\mathrm{g}}}-\hat{\varrho}{\tilde{v_{\mathrm{g}}}}^{2}-2 \hat{m}_{\mathrm{g}} \hat{m}_{\ell} \tilde{v}_{\ell} \hat{\mu}_{\mathrm{g}}-2 \hat{\zeta} \hat{m}_{\ell} \hat{v}_{\mathrm{g}} \tilde{v}_{\ell}, \\
& \hat{a}_{32}=\hat{\kappa} \hat{\varrho} \hat{\rho}_{\mathrm{g}}+2 \hat{m}_{\mathrm{g}} \hat{m}_{\ell}{\tilde{v_{\mathrm{g}}}}_{\mu_{\ell}}-2 \hat{m}_{\mathrm{g}} \tilde{v} \mathrm{~g}_{v_{\ell}}+2 \hat{m}_{\mathrm{g}} \tilde{v}_{\ell} \hat{v}_{\ell}-2 \hat{m}_{\mathrm{g}} \hat{m}_{\ell} \tilde{v}_{\ell} \hat{\mu}_{\ell}-\hat{\varrho} \tilde{v}_{\ell}^{2}
\end{aligned}
$$

and

obtained by the arithmetic averages

$$
\hat{\varrho}=\hat{m}_{\mathrm{g}}+\hat{\zeta} \hat{m}_{\ell}
$$

$$
\begin{aligned}
\hat{m_{\mathrm{g}}} & =\frac{1}{2}\left(m_{\mathrm{g}}^{\mathrm{L}}+m_{\mathrm{g}}^{\mathrm{R}}\right) \\
\hat{m}_{\ell} & =\frac{1}{2}\left(m_{\ell}^{\mathrm{L}}+m_{\ell}^{\mathrm{R}}\right) \\
\hat{v}_{\mathrm{g}} & =\frac{1}{2}\left(v_{\mathrm{g}}^{\mathrm{L}}+v_{\mathrm{g}}^{\mathrm{R}}\right) \\
\hat{v_{\ell}} & =\frac{1}{2}\left(v_{\ell}^{\mathrm{L}}+v_{\ell}^{\mathrm{R}}\right) \\
\hat{\rho_{\mathrm{g}}} & =\frac{1}{2}\left(\rho_{\mathrm{g}}^{\mathrm{L}}+\rho_{\mathrm{g}}^{\mathrm{R}}\right) \\
\hat{\rho_{\ell}} & =\frac{1}{2}\left(\rho_{\ell}^{\mathrm{L}}+\rho_{\ell}^{\mathrm{R}}\right),
\end{aligned}
$$

as well as the Roe-type averages

$$
\begin{aligned}
& \tilde{v}_{\mathrm{g}}=\frac{\sqrt{m_{\mathrm{g}}^{\mathrm{L}}} v_{\mathrm{g}}^{\mathrm{L}}+\sqrt{m_{\mathrm{g}}^{\mathrm{R}}} v_{\mathrm{g}}^{\mathrm{R}}}{\sqrt{m_{\mathrm{g}}^{\mathrm{L}}}+\sqrt{m_{\mathrm{g}}^{\mathrm{R}}}} \\
& \tilde{v}_{\ell}=\frac{\sqrt{m_{\ell}^{\mathrm{L}}} v_{\ell}^{\mathrm{L}}+\sqrt{m_{\ell}^{\mathrm{R}}} v_{\ell}^{\mathrm{R}}}{\sqrt{m_{\ell}^{\mathrm{L}}}+\sqrt{m_{\ell}^{\mathrm{R}}}},
\end{aligned}
$$


and where $\hat{\kappa}$ is obtained as described in Section 2.2.6, satisfies the Roe conditions R1 and R3 for the drift-flux model described in Section 1, provided that the Roe-averaged slip derivatives $\hat{\mu}_{\mathrm{g}}, \hat{\mu}_{\ell}$ and $\hat{\mu_{\mathrm{v}}} \equiv 1-\hat{\zeta}$ satisfy

$$
\hat{\mu_{\mathrm{g}}}\left(m_{\mathrm{g}}^{\mathrm{R}}-m_{\mathrm{g}}^{\mathrm{L}}\right)+\hat{\mu_{\ell}}\left(m_{\ell}^{\mathrm{R}}-m_{\ell}^{\mathrm{L}}\right)+\hat{\mu_{\mathrm{v}}}\left(v_{\mathrm{g}}^{\mathrm{R}}-v_{\mathrm{g}}^{\mathrm{L}}\right)=\Phi^{\mathrm{R}}-\Phi^{\mathrm{L}} .
$$

Furthermore, following the discussions of Sections 2.2.7 and 2.3, we make the following definitions:

Definition 1. The matrix $\hat{\boldsymbol{A}}$ described by Proposition 2, used in conjunction with the averages $\hat{\mu}_{\mathrm{g}}, \hat{\mu_{\ell}}$ and $\hat{\mu_{\mathrm{v}}}$ described by (115)-(117), satisfies the Roe conditions R1 and R3 for the drift-flux model supplied with a general, sufficiently smooth slip relation $\Phi$. The Roe scheme obtained by solving the linearized Riemann problem defined by this matrix $\hat{\boldsymbol{A}}$ will in the following be termed the RoeGen scheme.

Definition 2. The matrix $\hat{\boldsymbol{A}}$ described by Proposition 2 , used in conjunction with the averages $\hat{\mu}_{\mathrm{g}}, \hat{\mu_{\ell}}$ and $\hat{\mu_{\mathrm{v}}}$ described in Section 2.3, satisfies the Roe conditions R1 and R3 for the drift-flux model supplied with a Zuber-Findlay type slip relation $\Phi$, as expressed by (119). The Roe scheme obtained by solving the linearized Riemann problem defined by this matrix $\hat{\boldsymbol{A}}$ will in the following be termed the RoeZF scheme.

Remark 7. We have not been able to obtain explicit conditions under which RoeGen and RoeZF satisfy the condition R2. The numerical evidence indicates that if $\boldsymbol{q}^{\mathrm{L}}$ and $\boldsymbol{q}^{\mathrm{R}}$ are in the hyperbolic region of the model (see App. A), the RoeGen and RoeZF solvers tend to produce real eigenvalues and a hyperbolic linearization. No instance of complex eigenvalues, i.e. violation of R2, occurred for any calculations performed in the preparation of this paper.

\section{NumericAl Algorithm}

The present section provides a brief overview of the employed numerical algorithm, which is based on the wave-propagation (flux-difference splitting) form of Godunov's method presented by LeVeque ([16], Chap. 15). We start by giving a description of the general numerical method, and proceed with an explanation of how it relates to the Roe scheme.

\subsection{Framework}

A "high-resolution" extension of Godunov's method can be written as

$$
\boldsymbol{Q}_{i}^{n+1}=\boldsymbol{Q}_{i}^{n}-\frac{\Delta t}{\Delta x}\left(\mathcal{A}^{-} \Delta \boldsymbol{Q}_{i+1 / 2}+\mathcal{A}^{+} \Delta \boldsymbol{Q}_{i-1 / 2}\right)-\frac{\Delta t}{\Delta x}\left(\tilde{\boldsymbol{F}}_{i+1 / 2}-\tilde{\boldsymbol{F}}_{i-1 / 2}\right),
$$

where $\boldsymbol{Q}_{i}^{n}$ denotes the numerical approximation to the cell average of the vector of unknowns $\boldsymbol{q}\left(x, t_{n}\right)$ in control volume $i$ at time step $n$. The symbol $\mathcal{A}^{-} \Delta \boldsymbol{Q}_{i+1 / 2}$ denotes the net effect of all left-going waves at $x_{i+1 / 2}$, that is, at the control-volume boundary midway between $x_{i}$ and $x_{i+1}$, while $\mathcal{A}^{+} \Delta \boldsymbol{Q}_{i-1 / 2}$ measures the net effect of all right-going waves at $x_{i-1 / 2}$. The waves and wave speeds from the approximate Riemann solution are used to define

$$
\begin{aligned}
& \mathcal{A}^{-} \Delta \boldsymbol{Q}_{i-1 / 2}=\sum_{p=1}^{m}\left(s_{i-1 / 2}^{p}\right)^{-} \mathcal{W}_{i-1 / 2}^{p}, \\
& \mathcal{A}^{+} \Delta \boldsymbol{Q}_{i-1 / 2}=\sum_{p=1}^{m}\left(s_{i-1 / 2}^{p}\right)^{+} \mathcal{W}_{i-1 / 2}^{p},
\end{aligned}
$$

where $\mathcal{W}_{i-1 / 2}^{p}$ is the $p$ th wave arising in the solution to the Riemann problem at $x_{i-1 / 2}$, that is, it is a vector with one component for each equation. Here, $m$ is the number of waves, and since we will be using a linearized Riemann solver, it is equal to the number of equations. Furthermore, $s_{i-1 / 2}^{p}$ is the wave speed of the $p$ th wave and

$$
\left(s_{i-1 / 2}^{p}\right)^{+}=\max \left(s_{i-1 / 2}^{p}, 0\right), \quad\left(s_{i-1 / 2}^{p}\right)^{-}=\min \left(s_{i-1 / 2}^{p}, 0\right) .
$$


The flux vector $\tilde{\boldsymbol{F}}_{i-1 / 2}$ is the higher-order correction. It is given by

$$
\tilde{\boldsymbol{F}}_{i-1 / 2}=\frac{1}{2} \sum_{p=1}^{m}\left|s_{i-1 / 2}^{p}\right|\left(1-\frac{\Delta t}{\Delta x}\left|s_{i-1 / 2}^{p}\right|\right) \widetilde{\mathcal{W}}_{i-1 / 2}^{p},
$$

where $\widetilde{\mathcal{W}}_{i-1 / 2}^{p}$ is a limited version of the wave $\mathcal{W}_{i-1 / 2}^{p}$. With the correction terms, the method approaches second order for smooth solutions.

In the present work, we have taken account of source terms by adding the term $\Delta t \boldsymbol{S}_{i}$ to the right-hand side of $(152)$.

\subsection{Considerations for the Roe solver}

As noted in Section 2, the Roe scheme defines an approximate Riemann solution by replacing the nonlinear problem

$$
\frac{\partial \boldsymbol{q}}{\partial t}+\frac{\partial}{\partial x} \boldsymbol{f}(\boldsymbol{q})=\mathbf{0}
$$

by a linearized problem defined locally at each cell interface;

$$
\frac{\partial \hat{\boldsymbol{q}}}{\partial t}+\hat{\boldsymbol{A}}_{i-1 / 2} \frac{\partial \hat{\boldsymbol{q}}}{\partial x}=\mathbf{0} .
$$

For the Roe solver, we have the interpretation that

$$
\mathcal{A}^{ \pm} \Delta \boldsymbol{Q}_{i-1 / 2}=\hat{A}_{i-1 / 2}^{ \pm}\left(\boldsymbol{Q}_{i}-\boldsymbol{Q}_{i-1}\right)
$$

Herein,

$$
\hat{\boldsymbol{A}}_{i-1 / 2}^{ \pm}=\hat{\boldsymbol{R}}_{i-1 / 2} \hat{\boldsymbol{\Lambda}}_{i-1 / 2}^{ \pm} \hat{\boldsymbol{R}}_{i-1 / 2}^{-1},
$$

where $\hat{\boldsymbol{R}}_{i-1 / 2}$ is the matrix having the right eigenvectors $\hat{\boldsymbol{r}}_{i-1 / 2}$ of $\hat{\boldsymbol{A}}_{i-1 / 2}$ as its columns, and $\hat{\boldsymbol{\Lambda}}_{i-1 / 2}^{+}$and $\hat{\boldsymbol{\Lambda}}_{i-1 / 2}^{-}$ are the diagonal matrices containing the positive and negative eigenvalues, respectively, of $\hat{\boldsymbol{A}}_{i-1 / 2}$. Further, to satisfy the condition R1, we must have that

$$
\hat{\boldsymbol{A}}_{i-1 / 2}\left(\boldsymbol{Q}_{i}-\boldsymbol{Q}_{i-1}\right)=\sum_{p=1}^{m} s_{i-1 / 2}^{p} \mathcal{W}_{i-1 / 2}^{p} .
$$

The approximate Riemann solution consists of $m$ waves proportional to the right eigenvectors $\hat{\boldsymbol{r}}_{i-1 / 2}$, propagating with speeds

$$
s_{i-1 / 2}^{p}=\hat{\lambda}_{i-1 / 2}^{p}
$$

given by the eigenvalues. The proportionality coefficients $\beta_{i-1 / 2}^{p}$ can be found by solving the linear system

$$
\boldsymbol{Q}_{i}-\boldsymbol{Q}_{i-1}=\sum_{p=1}^{m} \beta_{i-1 / 2}^{p} \hat{\boldsymbol{r}}_{i-1 / 2}^{p}
$$

and $\beta_{i-1 / 2}^{p}$ can be interpreted as wave strengths ([28], Sect. 2.3.3). The solution of the equation (162) is

$$
\boldsymbol{\beta}_{i-1 / 2}=\hat{\boldsymbol{R}}_{i-1 / 2}^{-1}\left(\boldsymbol{Q}_{i}-\boldsymbol{Q}_{i-1}\right)
$$

whence the waves can be found as

$$
\mathcal{W}_{i-1 / 2}^{p}=\beta_{i-1 / 2}^{p} \hat{\boldsymbol{r}}_{i-1 / 2}^{p}
$$


TABLE 1. Initial states in the pure rarefaction test problem.

\begin{tabular}{rccl}
\hline Quantity & symbol (unit) & left & right \\
\hline Gas volume fraction & $\alpha_{\mathrm{g}}(-)$ & 0.6 & 0.68 \\
Pressure & $p(\mathrm{MPa})$ & 1.66667 & 1.17647 \\
Gas velocity & $v_{\mathrm{g}}(\mathrm{m} / \mathrm{s})$ & 34.4233 & 50.0 \\
Liquid velocity & $v_{\ell}(\mathrm{m} / \mathrm{s})$ & 34.4233 & 50.0 \\
\hline
\end{tabular}

TABLE 2. Parameters employed in the pure rarefaction test problem.

\begin{tabular}{ccc}
\hline & $c_{k}(\mathrm{~m} / \mathrm{s})$ & $\rho_{k}^{\circ}\left(\mathrm{kg} / \mathrm{m}^{3}\right)$ \\
\hline gas $(\mathrm{g})$ & 100 & 0 \\
liquid $(\ell)$ & 1000 & 998.924 \\
\hline
\end{tabular}

\subsubsection{Eigenstructure and computational efficiency}

As noted in Appendix A, the eigenstructure of the Roe matrix $\hat{\boldsymbol{A}}_{i-1 / 2}$ is quite complicated, and it is generally difficult to obtain analytical expressions for the eigenvalues and eigenvectors. Hence, in the present work, the eigenstructure was found numerically. In our implementation, approximately $55 \%$ of the total computation time was spent on the numerical eigenstructure decomposition.

The computational efficiency of our current Roe solver is given more focus in a companion paper [18], where a comparison is made to the recently proposed MUSTA approach of Titarev and Toro [27].

\subsubsection{Entropy solution}

For transonic rarefactions, that is, when an eigenvalue $\lambda^{p}$ is negative to the left of the $p$-wave, $\mathcal{W}^{p}$, and positive to the right, a scheme using a linearized Riemann solver may converge to an unphysical solution, violating the entropy condition [20].

Several remedies are conceivable, e.g. using Harten's entropy fix [13]. However, for the calculations presented in the following, the problem of entropy-condition violations did not occur.

\section{Numerical Simulations}

In this section, we illustrate the ability of the Roe method to produce accurate and non-oscillatory results for some numerical benchmark problems, including non-linear slip laws and transition to near-single-phase flow. Furthermore, for the case of the Zuber-Findlay slip relation, we show that the general method for the drift-flux model derived in Section 2.2.7 and the specific method of Section 2.3 give identical results.

The method (152) can be shown to be total variation diminishing (TVD) for scalar problems under the restriction that the Courant-Friedrichs-Lewy (CFL) number be smaller than $1 / 2$ ([16], Sect. 12.8). The calculations presented here were thus run using a CFL number of $1 / 2$.

\subsection{Pure rarefaction}

The first test case is a Riemann problem constructed by Baudin et al. [2], and whose solution is a pure rarefaction. Baudin et al. took the liquid to have a constant density. Here, however, both phases are treated as compressible. The considered horizontal tube is $100 \mathrm{~m} \mathrm{long}$, and there is a jump in the initial state at $x=50 \mathrm{~m}$. The initial values are given in Table 1, and we use the equation of state (13) with parameters reported in Table 2.

In the present problem, the no-slip law is used, that is, $\Phi \equiv 0$. In this case, the Roe average derived for the Zuber-Findlay slip relation (Sect. 2.3), and the general Roe average (Sect. 2.2.7), give the same numerical scheme. 


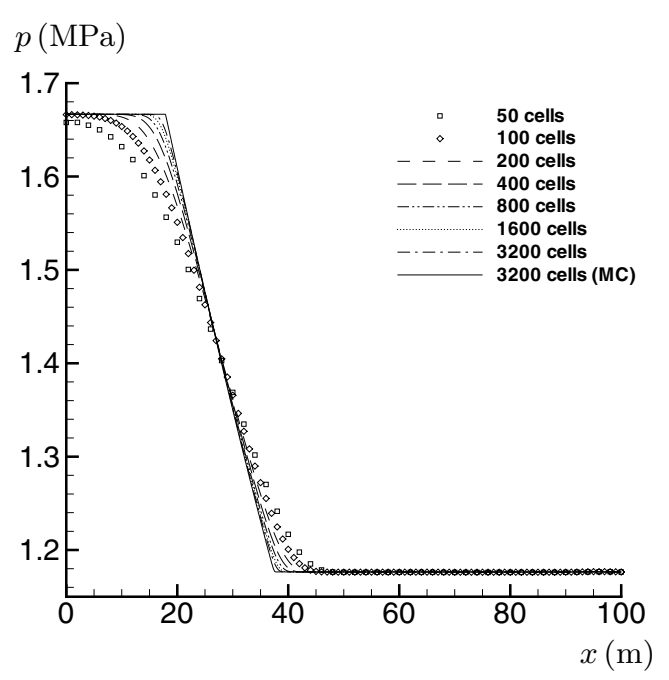

(a) No limiter (first-order)

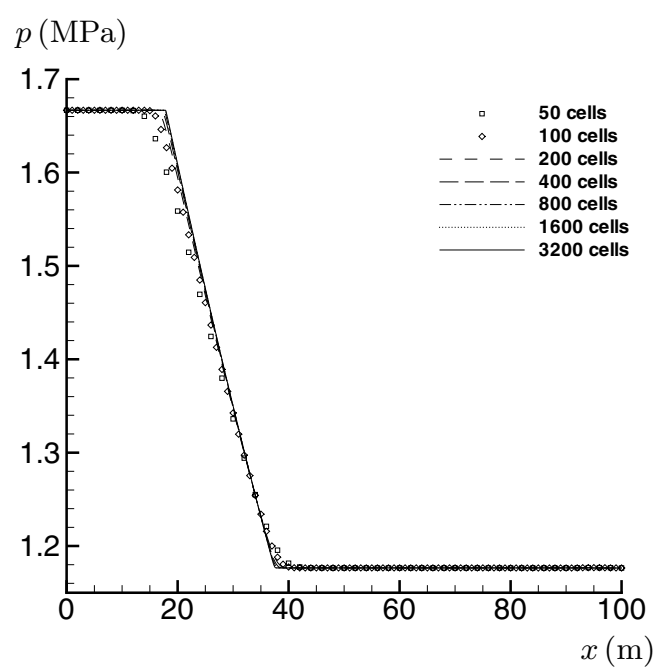

(b) Monotonized central difference (MC) limiter

Figure 1. Pressure for the pure rarefaction test problem. Convergence of the Roe method with and without a limiter function.

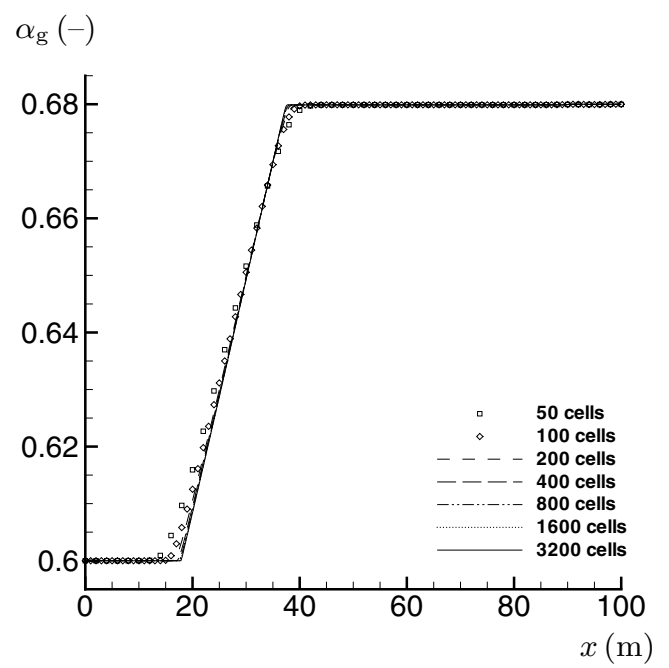

(a) Gas volume fraction

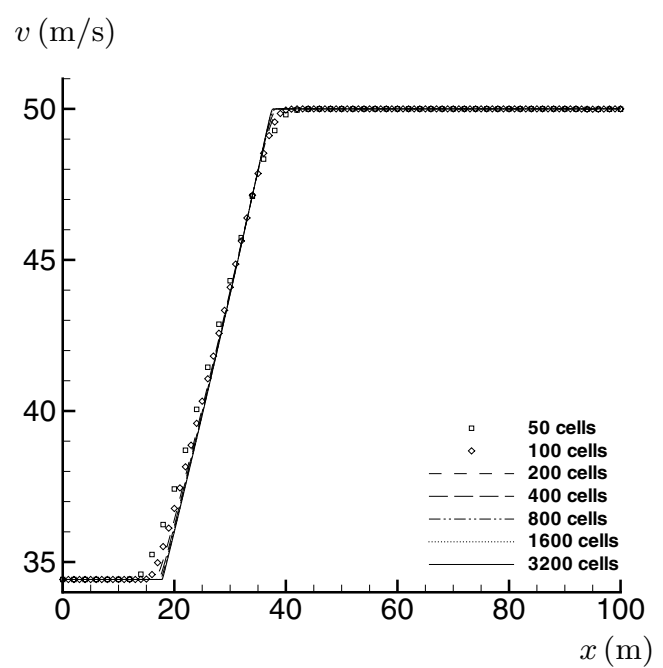

(b) Velocity

FiguRE 2. Gas volume fraction and velocity for the pure rarefaction test problem. Convergence of the Roe method using the MC limiter.

Pressure profiles at $t=0.8 \mathrm{~s}$ are presented for various grid sizes in Figure 1. Figure 1a shows the results obtained using the first-order scheme, that is, without the use of a limiter function, while in Figure 1b, the monotonized central-difference (MC) limiter [32] (see also [16], Sect. 6.11) has been employed. The first-order Roe scheme compares very well with the results presented in [2], and it can be seen that the use of the MC limiter provides an improved resolution of the rarefaction wave. The remaining physical variables are shown in Figure 2. 
TABLE 3. Initial states in the Shock Tube 1 problem.

\begin{tabular}{rccc}
\hline Quantity & symbol (unit) & left & right \\
\hline Gas volume fraction & $\alpha_{\mathrm{g}}(-)$ & 0.6 & 0.55 \\
Pressure & $p(\mathrm{kPa})$ & 522.825 & 803.959 \\
Gas velocity & $v_{\mathrm{g}}(\mathrm{m} / \mathrm{s})$ & 29.5138 & 2.5582 \\
Liquid velocity & $v_{\ell}(\mathrm{m} / \mathrm{s})$ & 24.7741 & 1.7372 \\
\hline
\end{tabular}

TABLE 4. Parameters employed in the Shock Tube 1 problem.

\begin{tabular}{ccc}
\hline & $c_{k}(\mathrm{~m} / \mathrm{s})$ & $\rho_{k}^{\circ}\left(\mathrm{kg} / \mathrm{m}^{3}\right)$ \\
\hline gas $(\mathrm{g})$ & 300 & 0 \\
liquid $(\ell)$ & 1000 & 999.196 \\
\hline
\end{tabular}

\subsection{Shock-tube problem 1}

We next consider a shock-tube problem where the solution consists of a 1-shock, a 2-contact and a 3-shock. This case was also studied by Baudin et al. [2] for the case of constant liquid density. The initial states can be found in Table 3, and the equation-of-state parameters are given in Table 4. The slip is given by the Zuber-Findlay relation (16) with $K=1.07$ and $S=0.2162$. Therefore, we employ the Roe average derived in Section 2.3 (RoeZF).

The convergence of the RoeZF scheme employing the MC limiter is shown in Figure 3, where the results are plotted at $t=0.5 \mathrm{~s}$. Both the shocks and the contact discontinuity are very sharply resolved.

\subsection{Shock-tube problem 2}

An alternative shock-tube problem has previously been studied by Evje and Fjelde [7] and Fjelde and Karlsen [11], for the case of constant liquid density. The initial states are given in Table 5, whereas Table 6 shows the equation-of-state parameters. In this problem, the Zuber-Findlay slip relation (16) is employed with $K=1.07$ and $S=0.216$.

Numerical results for grid refinement are displayed in Figure 4 for $t=1 \mathrm{~s}$. The solution at the shocks is non-oscillatory for all the variables, while the discontinuity is sharply resolved.

\subsection{Comparison of RoeGen and RoeZF}

In Section 2.3, we derived a Roe average specially for the Zuber-Findlay slip relation (RoeZF). Figure 5 shows numerical results for Shock Tube 1 obtained using RoeZF plotted on top of the solution calculated with the general Roe average (RoeGen) of Section 2.2.7. As can be seen, they are exactly the same. Figure 6 shows a similar comparison between RoeZF and RoeGen for Shock Tube 2. Again, the results are exactly the same. This gives confidence in the applicability of RoeGen for general slip relations.

\subsection{Pipe-flow problem}

The final test simulates a practical pipe-flow problem, and includes such challenges as a complex, non-linear slip relation and near-single-phase flow. The problem was introduced as Example 4 by Evje and Fjelde [8].

The equation-of-state parameters are given by Table 6 . In the slip relation (16), $K=1$ is constant, but $S$ is now a non-linear function of the volume fraction:

$$
S=S\left(\alpha_{\mathrm{g}}\right)=\frac{1}{2} \sqrt{1-\alpha_{\mathrm{g}}} .
$$

Further, a wall-friction model is included:

$$
F_{\mathrm{w}}=\frac{32 v_{\mathrm{m}} \eta_{\mathrm{m}}}{d^{2}}
$$




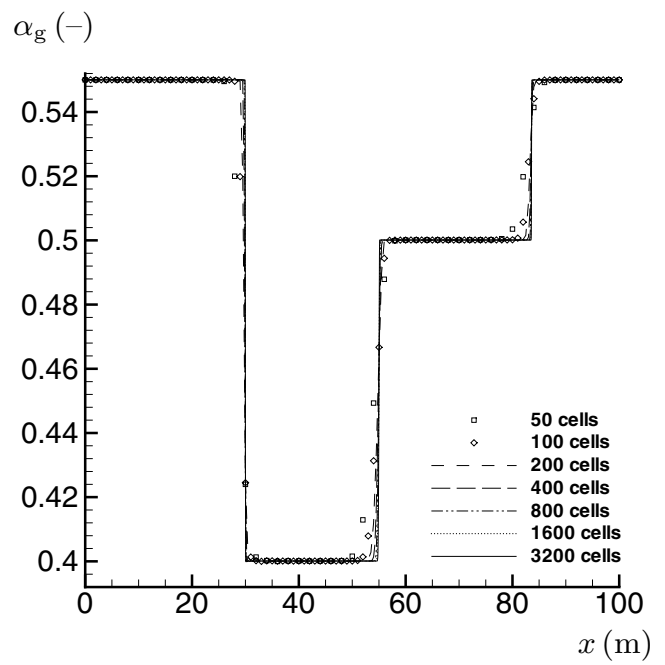

(a) Gas volume fraction

$v_{\mathrm{g}}(\mathrm{m} / \mathrm{s})$

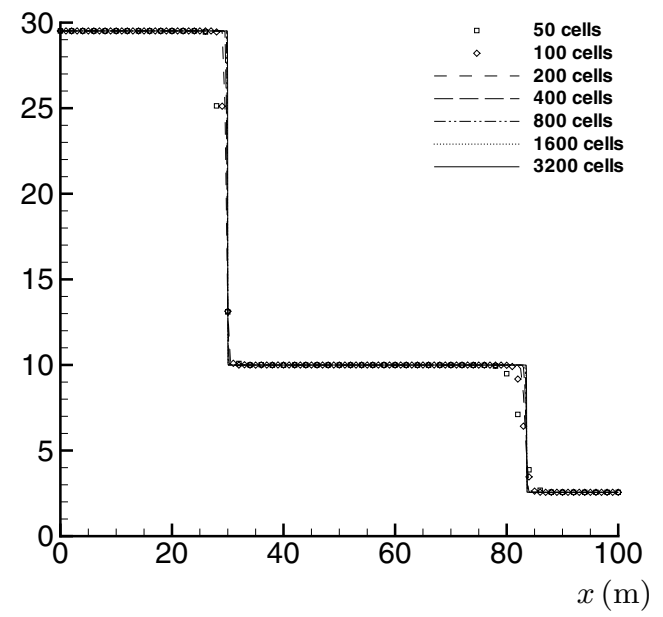

(c) Gas velocity

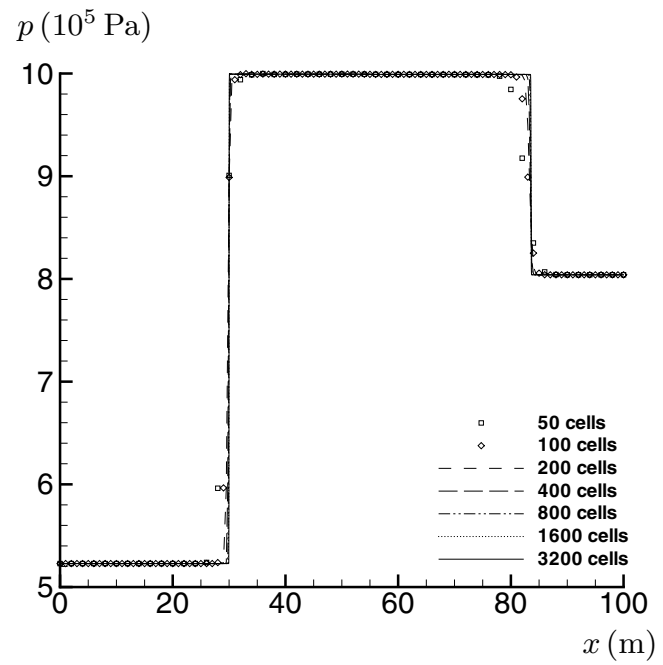

(b) Pressure

$v_{\ell}(\mathrm{m} / \mathrm{s})$

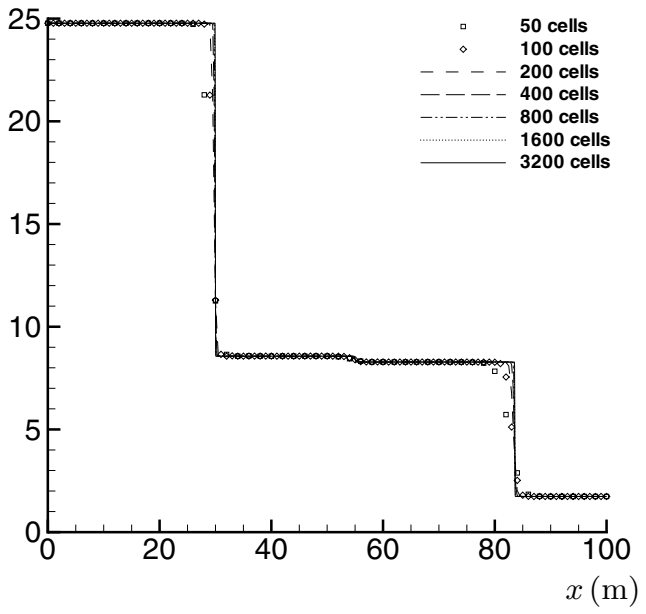

(d) Liquid velocity

Figure 3. Shock Tube 1. Convergence of the RoeZF method using the MC limiter.

where $v_{\mathrm{m}}$ is the mixture velocity,

$$
v_{\mathrm{m}}=\alpha_{\mathrm{g}} v_{\mathrm{g}}+\alpha_{\ell} v_{\ell},
$$

and the dynamic mixture viscosity, $\eta_{\mathrm{m}}$, is taken to be

$$
\eta_{\mathrm{m}}=\alpha_{\mathrm{g}} \eta_{\mathrm{g}}+\alpha_{\ell} \eta_{\ell}
$$

with $\eta_{\mathrm{g}}=5 \times 10^{-6} \mathrm{~Pa} \cdot \mathrm{s}$ and $\eta_{\ell}=5 \times 10^{-2} \mathrm{~Pa} \cdot \mathrm{s}$. 
TABLE 5. Initial states in the Shock Tube 2 problem.

\begin{tabular}{rccc}
\hline Quantity & symbol (unit) & left & right \\
\hline Gas volume fraction & $\alpha_{\mathrm{g}}(-)$ & 0.55 & 0.55 \\
Pressure & $p(\mathrm{kPa})$ & 80.450 & 24.282 \\
Gas velocity & $v_{\mathrm{g}}(\mathrm{m} / \mathrm{s})$ & 12.659 & 1.181 \\
Liquid velocity & $v_{\ell}(\mathrm{m} / \mathrm{s})$ & 10.370 & 0.561 \\
\hline
\end{tabular}

TABle 6. Parameters employed in the Shock Tube 2 and pipe-flow problems.

\begin{tabular}{ccc}
\hline & $c_{k}(\mathrm{~m} / \mathrm{s})$ & $\rho_{k}^{\circ}\left(\mathrm{kg} / \mathrm{m}^{3}\right)$ \\
\hline gas $(\mathrm{g})$ & $\sqrt{10^{5}}$ & 0 \\
liquid $(\ell)$ & 1000 & 999.9 \\
\hline
\end{tabular}

The problem consists of a horizontal pipe of length $l=1000 \mathrm{~m}$ and inner diameter $d=0.1 \mathrm{~m}$. Initially, it is filled with stagnant, almost-pure liquid, with $\alpha_{\mathrm{g}}=1 \times 10^{-5}$. Furthermore, the details of the simulation are specified as follows:

- The simulation lasts for $175 \mathrm{~s}$.

- Between $t=0$ and $t=10 \mathrm{~s}$, the gas and liquid inlet mass-flow rates are linearly increased from zero to $0.08 \mathrm{~kg} / \mathrm{s}$ and $12.0 \mathrm{~kg} / \mathrm{s}$, respectively.

- From $t=10 \mathrm{~s}$ to $t=175 \mathrm{~s}$, the inlet liquid mass-flow rate is kept constant.

- The inlet gas mass-flow rate is kept constant between $t=10 \mathrm{~s}$ and $t=50 \mathrm{~s}$.

- Between $t=50 \mathrm{~s}$ and $t=70 \mathrm{~s}$, the inlet gas mass-flow rate is linearly decreased from $0.08 \mathrm{~kg} / \mathrm{s}$ to $1 \times 10^{-8} \mathrm{~kg} / \mathrm{s}$, after which it is kept constant.

- At the outlet, the pressure is kept constant at $p=1 \times 10^{5} \mathrm{~Pa}$.

Calculations were performed using the RoeGen method. The physical variables are plotted in Figure 7 for various grids. It can be observed that for the 200-cell grid, the numerical solution is already close to the one obtained on fine grids. The present results compare favourably with those presented in [8]. Furthermore, one may note that the transition to near-single-phase flow is handled well.

\section{SUMMARY}

A quasi-linear formulation of the general drift-flux model, describing the flow of two-phase mixtures in pipelines, has been presented. Based on this formulation, a linearized Riemann solver of the type proposed by Roe has been derived.

The complexity of the closure laws inherent in the model prevents us from using the parameter-vector strategy originally proposed by Roe. Instead, we satisfy the Roe conditions using alternative strategies, enabling us to

- split the problem into independently solvable parts;

- handle general formulations of the closure laws within a single framework.

Hence, we are able to construct a genuine Roe scheme purely by algebraic manipulation of the flux Jacobian.

Even for the most general case, our proposed linearized Riemann solver is to a large extent constructed from arithmetic averages; it is consequently relatively efficient. Numerical examples have been presented, illustrating that the solver possesses the accuracy and robustness properties one may expect from a Roe-type method.

The approach here presented may be relevant for other systems of conservation laws where the flux vector is only partially available in algebraic form. 


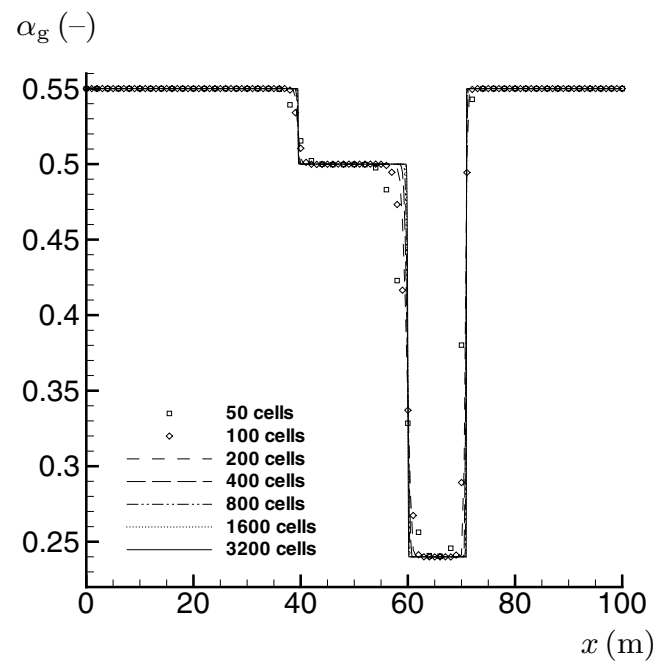

(a) Gas volume fraction

$v_{\mathrm{g}}(\mathrm{m} / \mathrm{s})$

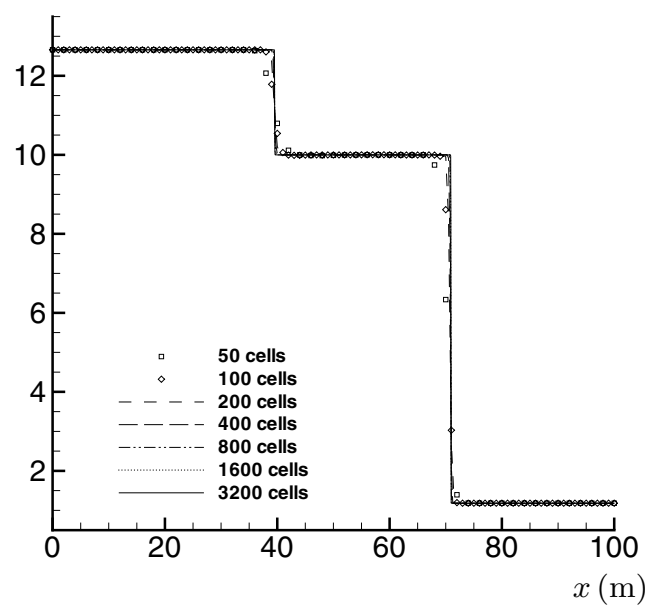

(c) Gas velocity

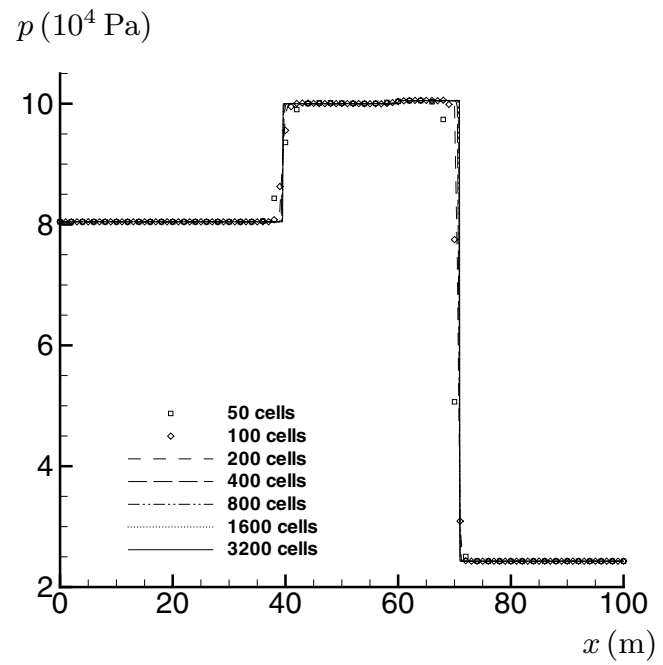

(b) Pressure

$v_{\ell}(\mathrm{m} / \mathrm{s})$

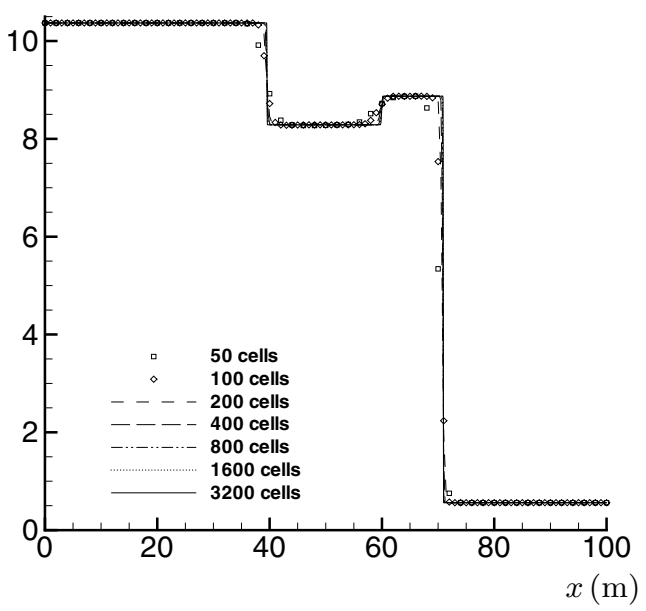

(d) Liquid velocity

Figure 4. Shock Tube 2. Convergence of the RoeZF method using the MC limiter.

\section{Appendix A. Hyperbolicity}

By definition, the drift-flux model is hyperbolic provided the Jacobian matrix (45) is diagonalizable in the real numbers. The eigenvalue equation for (45) becomes

$$
\begin{aligned}
\left(\lambda-v_{\mathrm{g}}\right)\left(\lambda-v_{\ell}\right)\left(\varrho \lambda-m_{\mathrm{g}} v_{\mathrm{g}}-\zeta m_{\ell} v_{\ell}\right)+ & m_{\mathrm{g}} m_{\ell}\left(\mu_{\ell}\left(\lambda-v_{\mathrm{g}}\right)^{2}-\mu_{\mathrm{g}}\left(\lambda-v_{\ell}\right)^{2}\right) \\
& +\kappa \rho_{\mathrm{g}} \rho_{\ell}\left(\alpha_{\mathrm{g}} \alpha_{\ell}\left(\rho_{\mathrm{g}} \mu_{\mathrm{g}}-\rho_{\ell} \mu_{\ell}\right)-\alpha_{\mathrm{g}}\left(\lambda-v_{\ell}\right)-\zeta \alpha_{\ell}\left(\lambda-v_{\mathrm{g}}\right)\right)=0
\end{aligned}
$$

Although this third-order polynomial equation is formally solvable in the algebraic numbers, this does not yield tractable expressions and a simple hyperbolicity criterion with general validity is not known. 


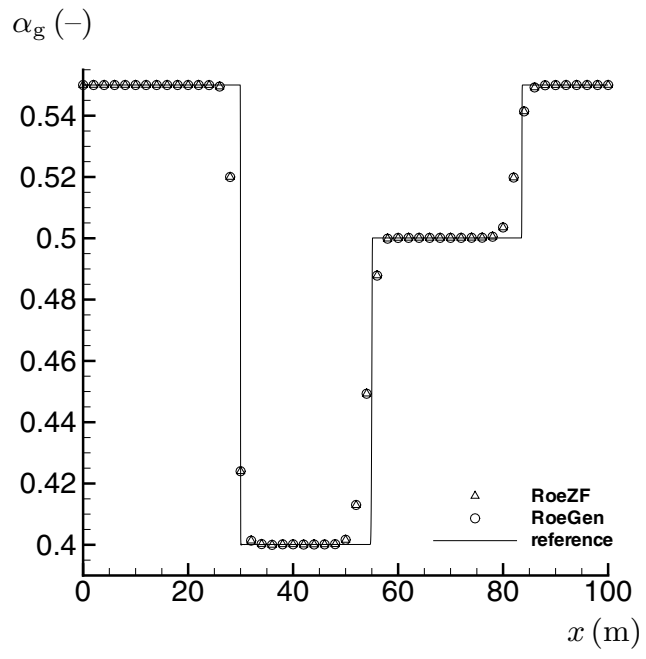

(a) Gas volume fraction

$v_{\mathrm{g}}(\mathrm{m} / \mathrm{s})$

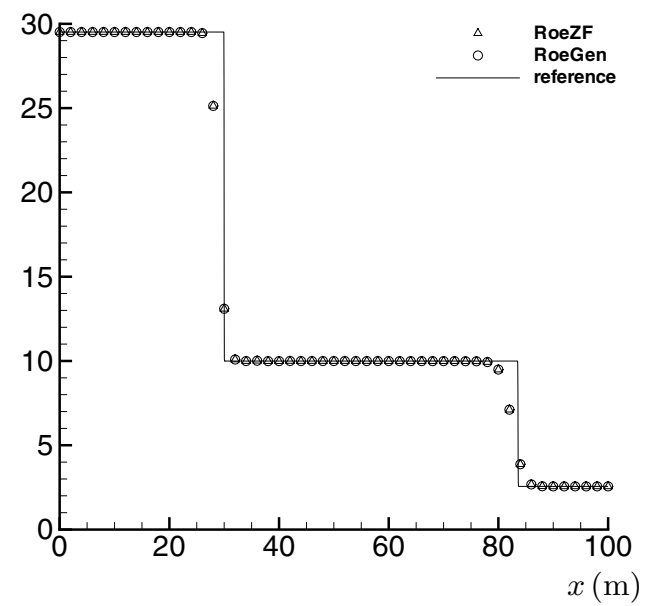

(c) Gas velocity

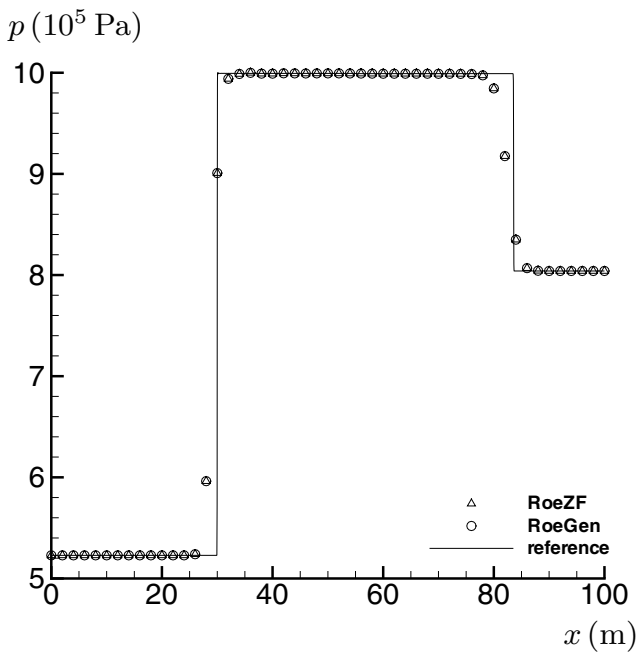

(b) Pressure

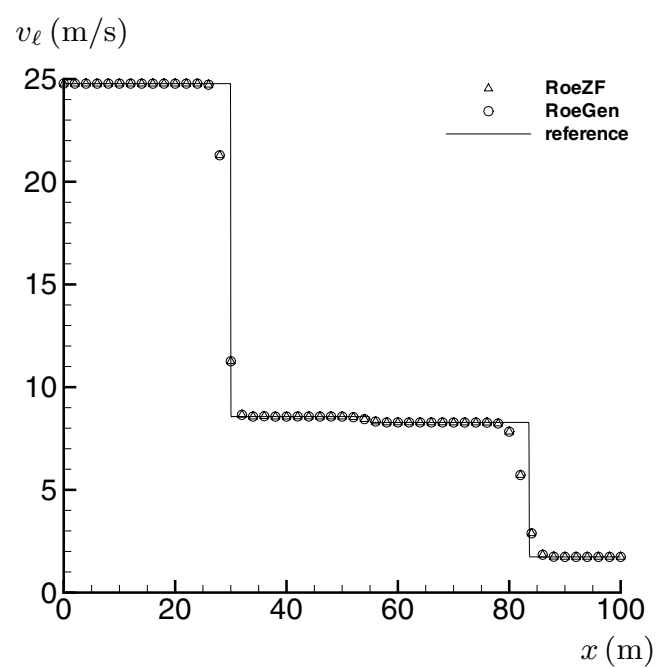

(d) Liquid velocity

Figure 5. Shock Tube 1. Comparison of the Zuber-Findlay average (RoeZF) and the general Roe-average (RoeGen) on a 50-cell grid, using the MC limiter. The 3200-cell solution is shown for reference.

However, we will present an approximate hyperbolicity condition valid for the Zuber-Findlay law, a result previously obtained by Benzoni-Gavage [5].

We assume incompressible liquid

$$
\frac{\partial \rho_{\ell}}{\partial p}=0
$$

as well as the Zuber-Findlay relation

$$
v_{\mathrm{g}}=K\left(\alpha_{\mathrm{g}} v_{\mathrm{g}}+\alpha_{\ell} v_{\ell}\right)+S .
$$




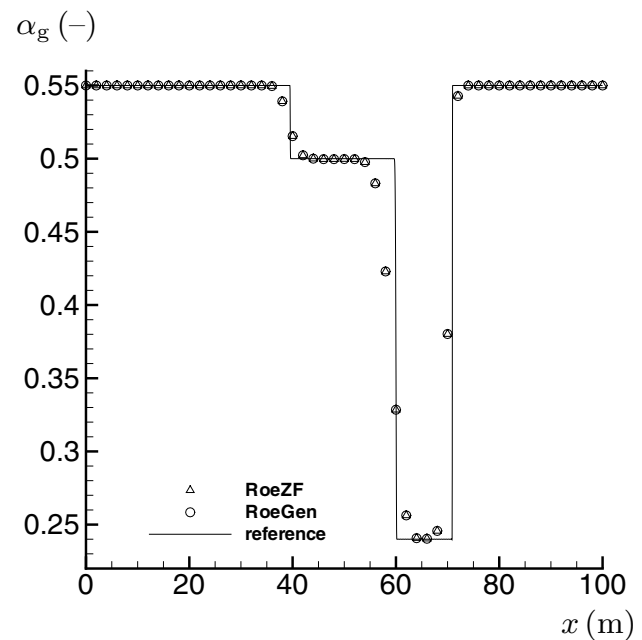

(a) Gas volume fraction

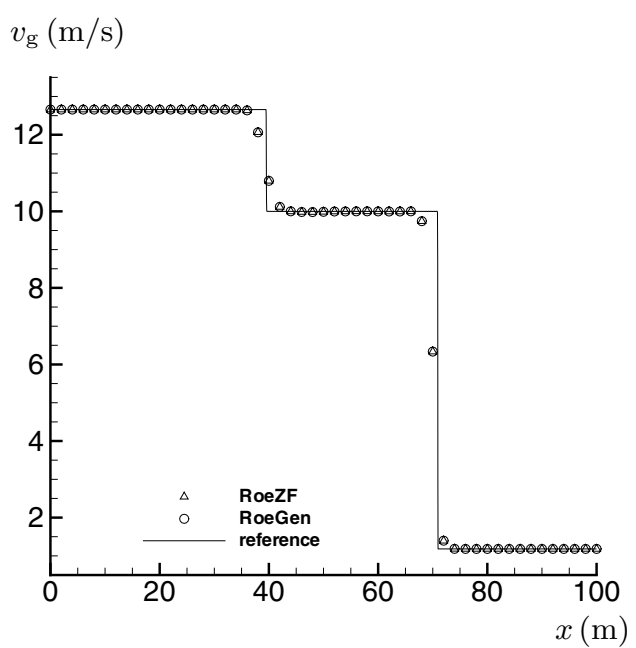

(c) Gas velocity

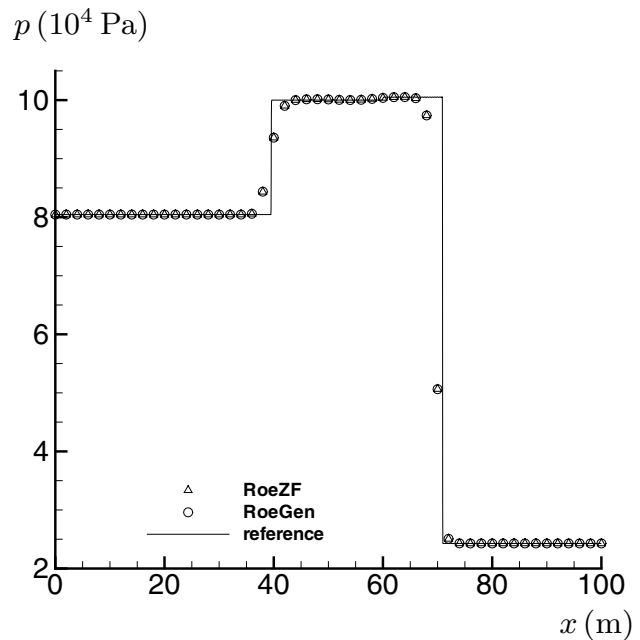

(b) Pressure

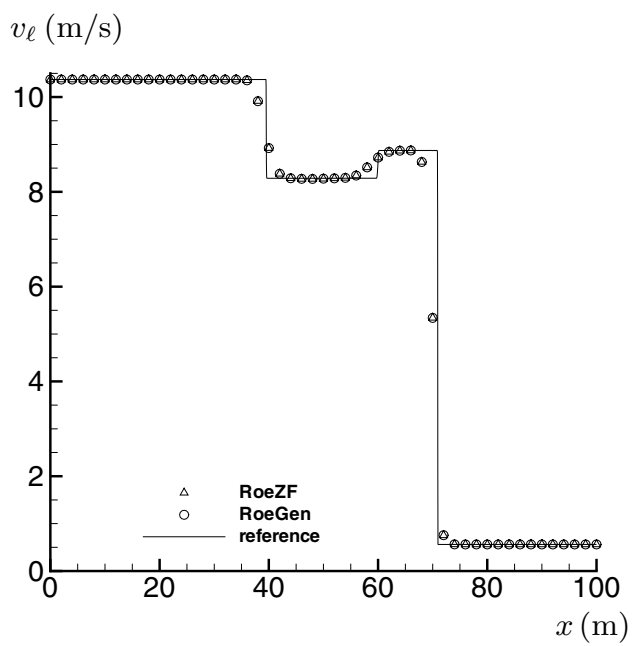

(d) Liquid velocity

Figure 6. Shock Tube 2. Comparison of the Zuber-Findlay average (RoeZF) and the general Roe-average (RoeGen) on a 50-cell grid, using the MC limiter. The 3200-cell solution is shown for reference.

Then from (121)-(122) we obtain

$$
\begin{aligned}
& \mu_{\mathrm{g}}=0 \\
& \mu_{\ell}=-\frac{v_{\mathrm{g}}-v_{\ell}}{m_{\ell}} .
\end{aligned}
$$

Inserting (172) and (173) into (169) we obtain

$$
\left(\lambda-v_{\mathrm{g}}\right)\left(\lambda-v_{\ell}\right)\left(\varrho \lambda-m_{\mathrm{g}} v_{\mathrm{g}}-\zeta m_{\ell} v_{\ell}\right)-m_{\mathrm{g}}\left(v_{\mathrm{g}}-v_{\ell}\right)\left(\lambda-v_{\mathrm{g}}\right)^{2}-\kappa \rho_{\mathrm{g}} \rho_{\ell}\left(\alpha_{\mathrm{g}}+\zeta \alpha_{\ell}\right)\left(\lambda-v_{\mathrm{g}}\right)=0
$$




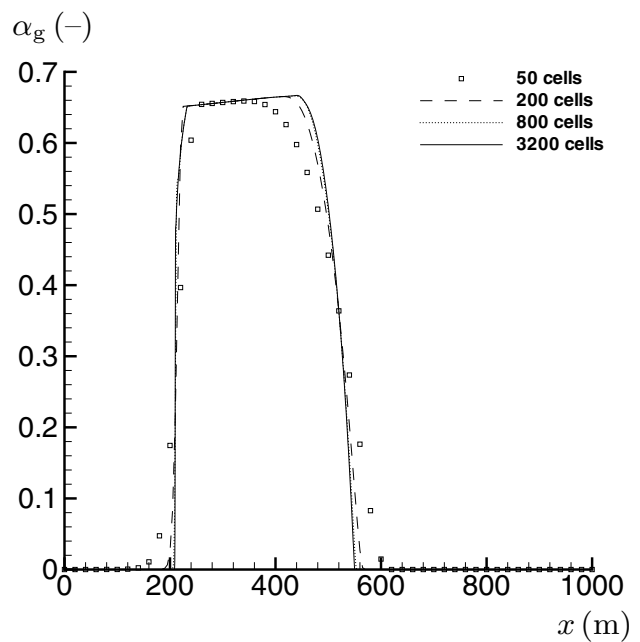

(a) Gas volume fraction

$v_{\mathrm{g}}(\mathrm{m} / \mathrm{s})$

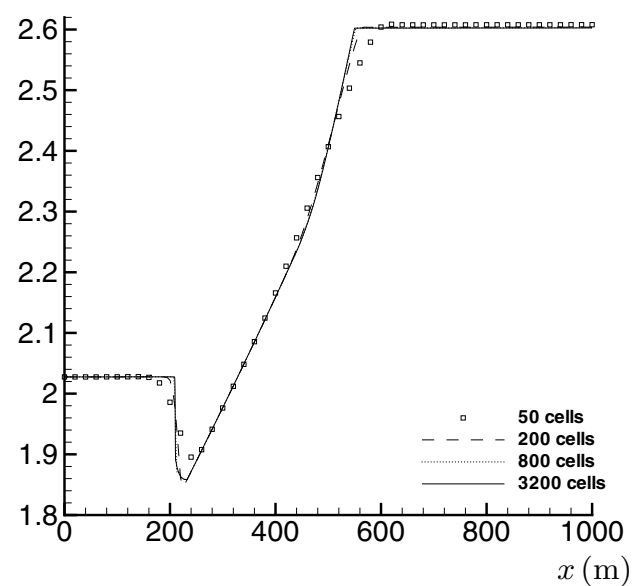

(c) Gas velocity

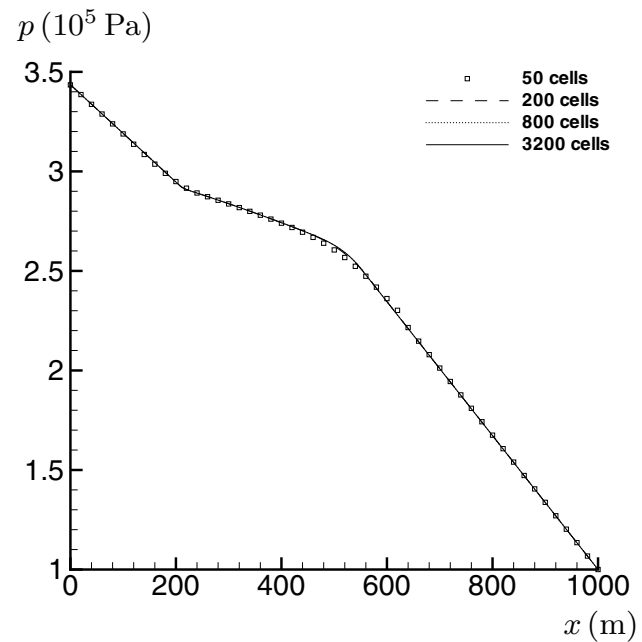

(b) Pressure

$v_{\ell}(\mathrm{m} / \mathrm{s})$

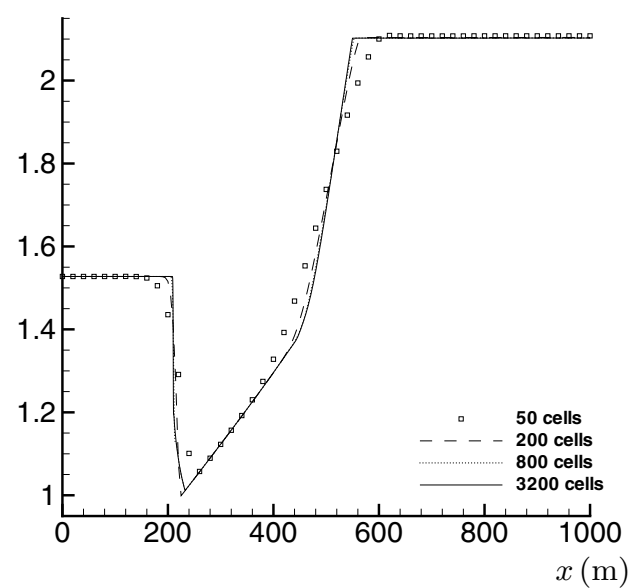

(d) Liquid velocity

Figure 7. Pipe-flow test problem. Convergence of the RoeGen method using the MC limiter.

This may be solved to yield the eigenvalues

$$
\lambda_{1}=v_{\mathrm{g}}, \quad \lambda_{2,3}=u \pm c
$$

where

$$
u=\frac{m_{\mathrm{g}} v_{\mathrm{g}}+\zeta m_{\ell} v_{\ell}}{m_{\mathrm{g}}+\zeta m_{\ell}}
$$

and

$$
c=\frac{\sqrt{\kappa \rho_{\mathrm{g}} \rho_{\ell}\left(\alpha_{\mathrm{g}}+\zeta \alpha_{\ell}\right)\left(m_{\mathrm{g}}+\zeta m_{\ell}\right)-\zeta m_{\ell} m_{\mathrm{g}}\left(v_{\mathrm{g}}-v_{\ell}\right)^{2}}}{m_{\mathrm{g}}+\zeta m_{\ell}} .
$$


Any loss of hyperbolicity must be associated with (177), from which we obtain the criterion

$$
\left(v_{\mathrm{g}}-v_{\ell}\right)^{2}<\kappa \frac{\alpha_{\mathrm{g}}+\zeta \alpha_{\ell}}{\alpha_{\mathrm{g}} \zeta \alpha_{\ell}}\left(m_{\mathrm{g}}+\zeta m_{\ell}\right)
$$

The criterion (178) is consequently an exact hyperbolicity criterion for the Zuber-Findlay law and incompressible liquid. Numerical investigations indicate that (178) may be a good approximation to the hyperbolicity criterion also for more general thermodynamic laws.

Acknowledgements. The authors thank the Research Council of Norway for financial support. We also thank the referees and editors, as well as our colleague Erik B. Hansen, for their valuable comments.

\section{REFERENCES}

[1] R. Abgrall and R. Saurel, Discrete equations for physical and numerical compressible multiphase mixtures. J. Comput. Phys. 186 (2003) 361-396.

[2] M. Baudin, C. Berthon, F. Coquel, R. Masson and Q.H. Tran, A relaxation method for two-phase flow models with hydrodynamic closure law. Numer. Math. 99 (2005) 411-440.

[3] M. Baudin, F. Coquel and Q.H. Tran, A semi-implicit relaxation scheme for modeling two-phase flow in a pipeline. SIAM J. Sci. Comput. 27 (2005) 914-936.

[4] K.H. Bendiksen, An experimental investigation of the motion of long bubbles in inclined tubes. Int. J. Multiphas. Flow 10 (1984) 467-483.

[5] S. Benzoni-Gavage, Analyse numérique des modèles hydrodynamiques d'écoulements diphasiques instationnaires dans les réseaux de production pétrolière. Thèse ENS Lyon, France (1991).

[6] J. Cortes, A. Debussche and I. Toumi, A density perturbation method to study the eigenstructure of two-phase flow equation systems, J. Comput. Phys. 147 (1998) 463-484.

[7] S. Evje and K.K. Fjelde, Hybrid flux-splitting schemes for a two-phase flow model. J. Comput. Phys. 175 (2002) 674-201.

[8] S. Evje and K.K. Fjelde, On a rough AUSM scheme for a one-dimensional two-phase model. Comput. Fluids 32 (2003) $1497-1530$.

[9] S. Evje and T. Flåtten, Hybrid flux-splitting schemes for a common two-fluid model. J. Comput. Phys. 192 (2003) 175-210.

[10] I. Faille and E. Heintzé, A rough finite volume scheme for modeling two-phase flow in a pipeline. Comput. Fluids 28 (1999) 213-241.

[11] K.K. Fjelde and K.H. Karlsen, High-resolution hybrid primitive-conservative upwind schemes for the drift-flux model. Comput. Fluids 31 (2002) 335-367.

[12] F. França and R.T. Lahey, Jr., The use of drift-flux techniques for the analysis of horizontal two-phase flows. Int. J. Multiphas. Flow 18 (1992) 787-801.

[13] A. Harten, High resolution schemes for hyperbolic conservation laws. J. Comput. Phys. 49 (1983) 357-393.

[14] S. Jin and Z.P. Xin, The relaxation schemes for systems of conservation laws in arbitrary space dimensions. Commun. Pur. Appl. Math. 48 (1995) 235-276.

[15] S. Karni, E. Kirr, A. Kurganov and G. Petrova, Compressible two-phase flows by central and upwind schemes. ESAIM: M2AN 38 (2004) 477-493.

[16] R.J. LeVeque, Finite Volume Methods for Hyperbolic Problems. Cambridge University Press, Cambridge, UK (2002).

[17] J.M. Masella, Q.H. Tran, D. Ferre and C. Pauchon, Transient simulation of two-phase flows in pipes. Int. J. Multiphas. Flow 24 (1998) 739-755.

[18] S.T. Munkejord, S. Evje and T. Flåtten, The multi-stage centred-scheme approach applied to a drift-flux two-phase flow model. Int. J. Numer. Meth. Fl. 52 (2006) 679-705.

[19] A. Murrone and H. Guillard, A five equation reduced model for compressible two phase flow problems. J. Comput. Phys. 202 (2005) 664-698.

[20] S. Osher, Riemann solvers, the entropy condition, and difference approximations. SIAM J. Numer. Anal. 21 (1984) $217-235$.

[21] V.H. Ransom and D.L. Hicks, Hyperbolic two-pressure models for two-phase flow. J. Comput. Phys. 53 (1984) $124-151$.

[22] P.L. Roe, Approximate Riemann solvers, parameter vectors, and difference schemes. J. Comput. Phys. 43 (1981) 357-372.

[23] J.E. Romate, An approximate Riemann solver for a two-phase flow model with numerically given slip relation. Comput. Fluids 27 (1998) 455-477.

[24] L. Sainsaulieu, Finite volume approximation of two-phase fluid flow based on an approximate Roe-type Riemann solver. $J$. Comput. Phys. 121 (1995) 1-28. 
[25] R. Saurel and R. Abgrall, A multiphase Godunov method for compressible multifluid and multiphase flows. J. Comput. Phys. 150 (1999) 425-467.

[26] H.B. Stewart and B. Wendroff, Review article; Two-phase flow: models and methods. J. Comput. Phys. 56 (1984) $363-409$.

[27] V.A. Titarev and E.F. Toro, MUSTA schemes for multi-dimensional hyperbolic systems: analysis and improvements. Int. J. Numer. Meth. Fl. 49 (2005) 117-147.

[28] E.F. Toro, Riemann solvers and numerical methods for fluid dynamics, 2nd edn. Springer-Verlag, Berlin (1999).

[29] I. Toumi, An upwind numerical method for two-fluid two-phase flow models. Nucl. Sci. Eng. 123 (1996) 147-168.

[30] I. Toumi and D. Caruge, An implicit second-order numerical method for three-dimensional two-phase flow calculations. Nucl. Sci. Eng. 130 (1998) 213-225.

[31] I. Toumi and A. Kumbaro, An approximate linearized Riemann solver for a two-fluid model. J. Comput. Phys. 124 (1996) $286-300$.

[32] B. van Leer, Towards the ultimate conservative difference scheme IV. New approach to numerical convection. J. Comput. Phys. 23 (1977) 276-299.

[33] N. Zuber and J.A. Findlay, Average volumetric concentration in two-phase flow systems. J. Heat Transfer 87 (1965) $453-468$.

To access this journal online: www.edpsciences.org 